\title{
Non-radial pulsation, rotation and outburst in the Be star $\omega$ Orionis from the MuSiCoS 1998 campaign ${ }^{\star}$
}

\author{
C. Neiner ${ }^{1,2}$, A.-M. Hubert ${ }^{1}$, M. Floquet ${ }^{1}$, S. Jankov ${ }^{3,4}$, H. F. Henrichs ${ }^{2}$, B. Foing ${ }^{5}$, J. Oliveira ${ }^{5,6}$, \\ S. Orlando ${ }^{5,7}$, J. Abbott ${ }^{8,9}$, I. K. Baldry ${ }^{10}$, T. R. Bedding ${ }^{10}$, J. Cami ${ }^{2}$, H. Cao ${ }^{11}$, C. Catala ${ }^{12}$,
} K. P. Cheng ${ }^{13}$, A. Domiciano de Souza Jr ${ }^{14,3}$, E. Janot-Pacheco ${ }^{1,14}$, J. X. Hao ${ }^{11}$, L. Kaper ${ }^{2}$, A. Kaufer ${ }^{15}$, N. V. Leister ${ }^{14}$, J. E. Neff ${ }^{16}$, S. J. O’Toole ${ }^{10}$, D. Schäfer ${ }^{18}$, S. J. Smartt ${ }^{19,20}$, O. Stahl ${ }^{18}$, J. Telting ${ }^{8,21}$, S. Tubbesing ${ }^{18}$, and J. Zorec ${ }^{22}$

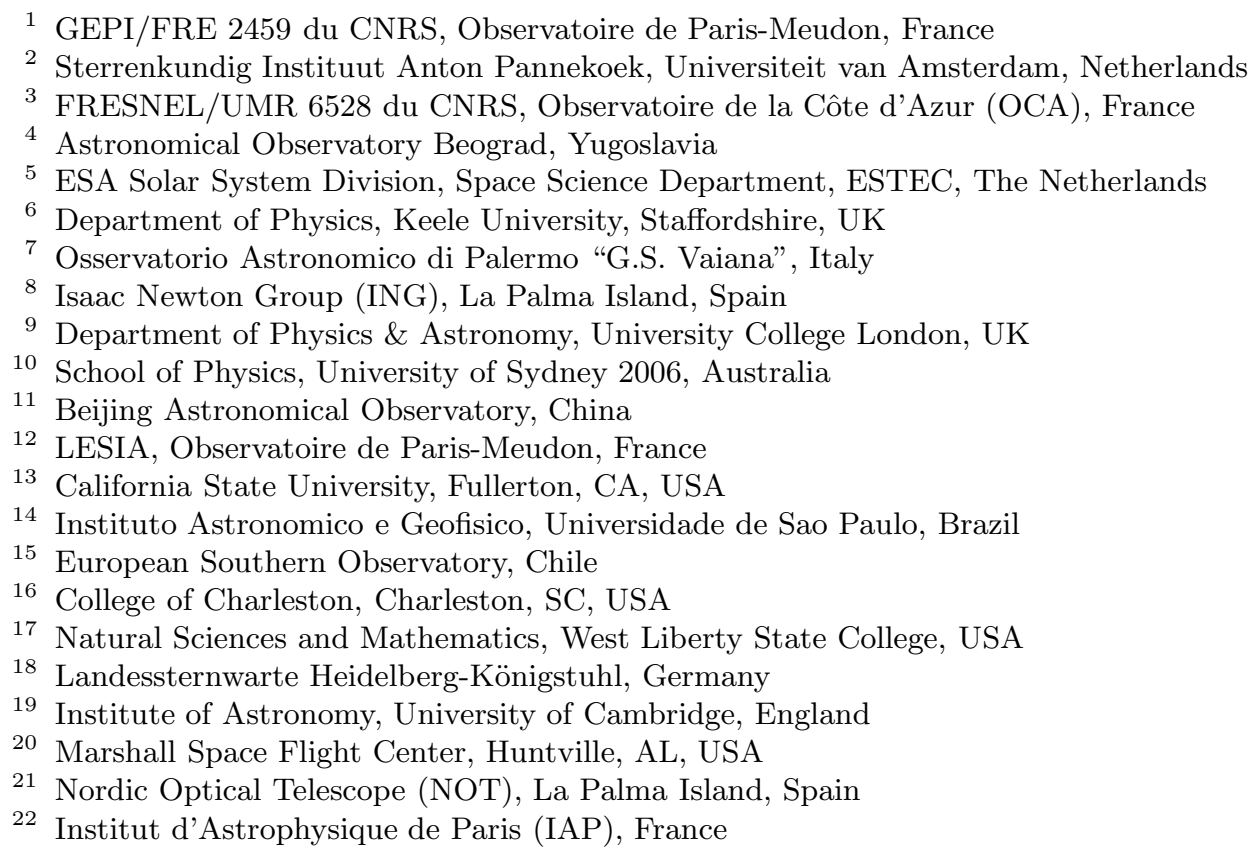

Received 27 December 2001 / Accepted 29 March 2002

\begin{abstract}
Ori (HD 37490, HR 1934) is a Be star known to have presented variations. In order to investigate the nature and origin of its short-term and mid-term variability, a study is performed of several spectral lines ( $\mathrm{H} \alpha, \mathrm{H} \delta, \mathrm{He}$ I $4471,4713,4921$, 5876, 6678, C II 4267, 6578, 6583, Mg II 4481, Si III 4553 and Si II 6347), based on 249 high signal-to-noise high-resolution spectra taken with 8 telescopes over 22 consecutive nights during the MuSiCoS (Multi SIte COntinuous Spectroscopy) campaign in November-December 1998. The stellar parameters are revisited and the projected rotational velocity $\left(v \sin i=179 \mathrm{~km} \mathrm{~s}^{-1}\right)$ is redetermined using several methods. With the MuSiCoS 98 dataset, a time series analysis of line-profile variations (LPVs) is performed using the Restricted Local Cleanest (RLC) algorithm and a least squares method. The behaviour of the velocity of the centroid of the lines, the equivalent widths and the apparent $v \sin i$ for several lines, as well as Violet and Red components of photospheric lines affected by emission (red He I lines, Si II 6347, C II 6578, 6583) are analyzed. The non-radial pulsation (NRP)
\end{abstract}

Send offprint requests to: C. Neiner, e-mail: Coralie.Neiner@obspm.fr

* Based on observations taken during the MuSiCoS 98 campaign at OHP (France), La Silla (ESO, Chile, ID 62.H-0270), Mount Stromlo (Australia), Xinglong Station (China), Kitt Peak (USA), MCT/LNA (Brazil) and INT (Isaac Newton Group, La Palma Island). 
model is examined using phase diagrams and the Fourier-Doppler Imaging (FDI) method. The LPVs are consistent with a NRP mode with $l=2$ or $3,|m|=2$ with frequency $1.03 \mathrm{~cd}^{-1}$. It is shown that an emission line outburst occurred in the middle of the campaign. Two scenarios are proposed to explain the behaviour of a dense cloud, temporarily orbiting around the star with a frequency $0.46 \mathrm{~cd}^{-1}$, in relation to the outburst.

Key words. stars: emission line, Be - stars: early-type - stars: individual: $\omega$ Ori - stars: oscillations - stars: activity line: profiles

\section{Introduction}

Be stars are non-supergiant B stars that at least once have displayed Balmer line emission. This occurs in about $20 \%$ of all B-type stars but, while this emission is thought to arise from a circumstellar disk, many details are unknown.

The early Be stars exhibit strongly variable winds evidenced by the rapidly variable UV resonance lines of highly ionized species, as well as spectral and photometric variations on timescales from hours to decades. The phases of emission in the optical and IR lines of hydrogen and several other species are called the Be phenomenon and most likely reflect changes in the structure of the circumstellar disk due to episodic ejections of mass.

The few interferometric observations of Be stars that exist (Stee \& Bittar 2001; Quirrenbach et al. 1997) show clear evidence for a non-spherical or flattened envelope, often thought to be related to the usually high rotational velocities of these stars (Harmanec 1982; Marlborough 1986). The stellar rotation rates, however, are always lower than the critical velocities at which the centrifugal force balances gravitation at the equator. Thus, the centrifugal force by itself is inadequate to explain the formation of a disk around these stars. Note that, although high rotational velocities are usually observed for Be stars, this property is not part of the definition of a Be star (Collins 1987), and some of them rotate slowly (e.g. $\beta$ Cep, Donati et al. 2001; see also Mennickent et al. 1994).

The key problems in understanding this phenomenon are then: (i) how to enhance the specific angular momentum of material so that it can be ejected and attain a stable orbit, and (ii) how to eject quantities of mass in a nonsteady fashion.

NRPs and magnetic field processes have been proposed as possible mechanisms to explain the Be phenomenon (e.g. Osaki 1986; Underhill 1987). Multiperiodicity has already been detected in LPV of Be stars and has been generally attributed to NRPs (e.g. Gies 1994; Rivinius et al. 1998a, hereafter R1). Up to now, only $\beta$ Cep is known to host a magnetic field (Henrichs et al. 2000a).

Although $\omega$ Ori has a rich history with suspected pulsation and variable wind properties with a period close to 1 day, its behavior has remained a mystery. For these reasons, and because it can be observed from both hemispheres, it is a very suitable target for multi-site observations. Therefore a MuSiCoS campaign on this star was organized in 1998. In this paper, we first discuss the stellar parameters of $\omega$ Ori (Sect. 2) and recall the observational history of this star (Sect. 3). The data obtained during the campaign are presented in Sect. 4 . In Sect. 5 we present a detailed analysis of the line-profile variations and give the frequencies found. The main frequency $f_{1}$ is studied in Sect. 6, while a second frequency $f_{2}$ is investigated in Sect. 7. Finally we discuss the obtained results (Sect. 8) and present concluding remarks (Sect. 9).

\section{Stellar parameters}

$\omega$ Ori has sometimes been classified as a Herbig Be star, (e.g. Thé et al. 1994) but the small IR excess is consistent with free-free emission rather than circumstellar dust (Hillenbrand et al. 1992). Moreover, it shows wind activity typical of classical Be stars of the same spectral type (Grady et al. 1996) and no X-ray emission has been detected for this star (Zinnecker \& Preibisch 1994). Therefore, $\omega$ Ori is considered as a classical Be star, as most researchers agree. Its radial velocity is $V_{\text {rad }}=$ $21.8 \mathrm{~km} \mathrm{~s}^{-1}$ (Duflot et al. 1995).

For an appropriate modeling of the rotational and pulsational phenomena, knowledge of the stellar parameters is essential. We compiled the most reliable data and attempted a critical evaluation of the various numbers using the usual astronomical formulae and the BCD (BarbierChalonge-Divan) spectrophotometric system (Zorec \& Briot 1991).

The distance to the star is not well constrained by Hipparcos parallax measurements (see Table 1), but much stronger limits are put by $\omega$ Ori's undisputed membership of the Orion OB1a association (e.g. Brown et al. 1994) located at $380 \pm 50 \mathrm{pc}$, which we adopt as the distance to the star.

One way to calculate the radius is to estimate the effective temperature, $T_{\text {eff }}$, from the spectral type and the luminosity $L$ from the visual magnitude, distance, extinction and bolometric correction (see Table 1). The spectral type as given by several sources varies between B2IIIe, B3IIIe and B2IVe. Ballereau et al. (1995) gives B2-3IIIe, which we adopt here.

$T_{\text {eff }}$ and $\log g$ are determined with the BCD method by deriving the photospheric spectrophotometric BCD parameters of the star $\left(\lambda_{1}, D_{*}\right)$. $\lambda_{1}$ gives the mean spectral position of the Balmer discontinuity and is a sensitive indicator of the stellar surface gravity, whereas $D_{*}$ is a measure of the Balmer jump and a diagnostic of the stellar effective temperature. With these two parameters, we can determine the MK spectral type, the absolute magnitude $M_{V}$ and the absolute bolometric magnitude $M_{\mathrm{bol}}$. 
Table 1. Stellar parameters for $\omega$ Ori (HD 37490, HR 1934). First column: derived from the usual astrophysical formulae. Second column: derived from the BCD (Barbier-ChalongeDivan spectrophotometric system) classification taking into account rapid rotational and evolutionary effects, with formal errors.

\begin{tabular}{lll}
\hline \hline & & BCD \\
Spectral Type & B2-3IIIe & B2III \\
$V_{\text {rad }}\left(\mathrm{km} \mathrm{s}^{-1}\right)$ & 21.8 & \\
$V$ & $4.55 \pm 0.1$ & 4.58 \\
$B-V$ & $-0.1 \pm 0.01$ & \\
$A_{V}$ & $0.32 \pm 0.05$ & \\
$D_{\text {Hipparcos }}(\mathrm{pc})$ & $500_{-330}^{+570}$ & \\
$D_{\text {OriOB1a }}(\mathrm{pc})$ & $380 \pm 50$ & $370 \pm 58$ \\
& & \\
$M_{V}$ & $-3.67 \pm 0.39$ & $-3.43 \pm 0.28$ \\
$M_{\text {bol }}$ & $-5.57 \pm 0.49$ & $-5.3 \pm 0.37$ \\
$\log \left(L / L_{\odot}\right)$ & $4.12 \pm 0.20$ & $4.03 \pm 0.15$ \\
$\log T_{\text {eff }}$ & & $4.306 \pm 0.016$ \\
$\log g$ & & $3.48 \pm 0.03$ \\
$R / R_{\odot}$ & $9.35_{-6.89}^{+12.66}$ & $6.84 \pm 0.25$ \\
$M / M_{\odot}$ & $9.91_{-5.04}^{+19.50}$ & $8.02 \pm 0.25$ \\
$v_{\text {crit }}\left(\mathrm{km} \mathrm{s}^{-1}\right)$ & $450_{-373}^{+542}$ & \\
$i\left(^{\circ}\right)$ & $35_{-25}^{+52}$ & $32 \pm 15$ \\
$v \sin i\left(\mathrm{~km} \mathrm{~s}^{-1}\right)$ & $179 \pm 4$ & $175 \pm 20$ \\
$f_{\text {rot }}\left(\mathrm{c} \mathrm{d}^{-1}\right)$ & $0.66_{-0.48}^{+0.91}$ & $0.79_{-0.49}^{+1.65}$ \\
\hline & &
\end{tabular}

They all fit with the parameters determined earlier (see Table 1). This method also gives the effective temperature $\log T_{\text {eff }}=4.306 \pm 0.016$ and the surface gravity $\log g=3.48 \pm 0.03$.

Below we argue that the most likely value of $v \sin i$ is $179 \mathrm{~km} \mathrm{~s}^{-1}$ (see Sect. 5.3). For such a star several corrections would apply, in particular in relation to the rotational deformation of the star and gravity darkening. Therefore the established parameters represent an average photosphere which corresponds to the observed hemisphere of the star deformed by rotation. The real parameters of the star are related to these observed parameters by functions of the stellar mass, angular velocity ratio $\omega$, inclination angle $i$ of the rotational axis and stellar age (Zorec et al. 2002, see also Sect. 2 in Floquet et al. 2000a).

These relations are solved using the evolutionary tracks of Schaller et al. (1992). We obtain $\omega=0.83 \omega_{\text {c }}$, where $\omega_{\mathrm{c}}$ is the critical angular velocity, and $i=32^{\circ} \pm 15$. This yields $R_{\mathrm{e}}=8.24 R_{\odot}$ for the equatorial radius and $M=8.02 M_{\odot}$, for the mass, implying a mean radius of $R=6.84 R_{\odot}$. The radius and mass derived from the usual formulae are given in Table 1 and agree with the ones derived from the BCD classification within the errors. We can compute the critical velocity $v_{\text {crit }}$ at which the star

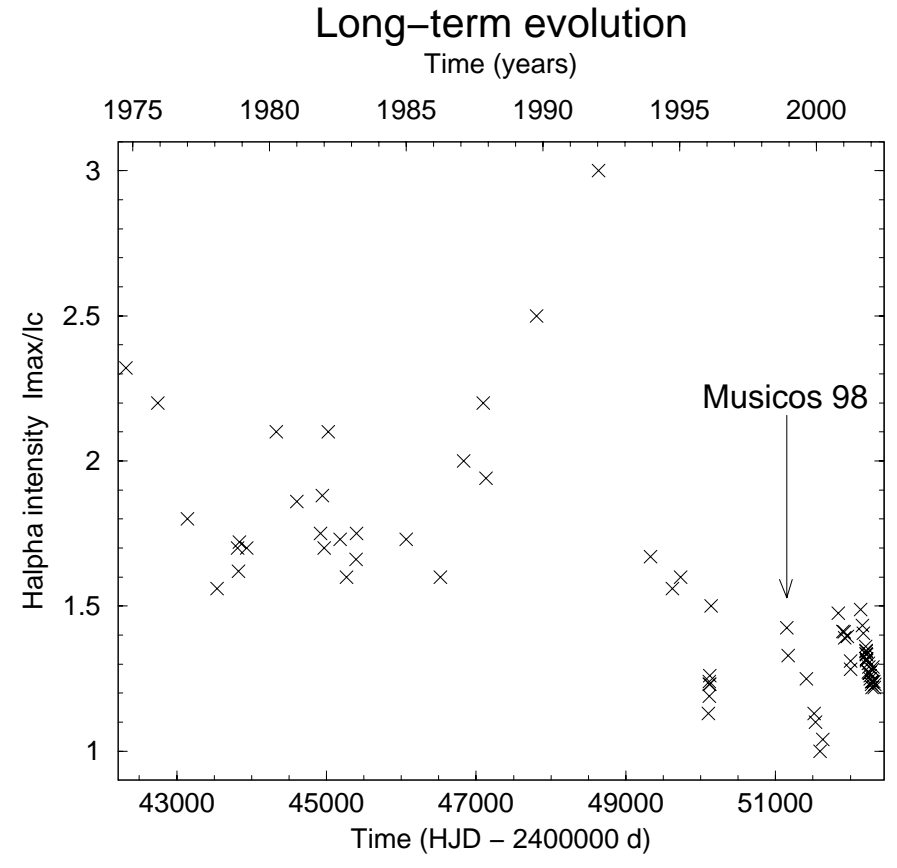

Fig. 1. Long-term evolution of the normalized $\mathrm{H} \alpha$ intensity of $\omega$ Ori. Data are taken from Andrillat \& Fehrenbach (1982), Balona et al. (2001), Banerjee et al. (2000), Bopp \& Dempsey (1989), Buil (2001), Dachs et al. (1981), Doazan et al. (1991), Hanuschik et al. (1996), Oudmaijer \& Drew (1999), Sonneborn et al. (1988), Srinivasan (1996), this paper and unpublished data obtained from TBL (France) in 2000, NTT (ESO) and $1.52 \mathrm{~m} /$ FEROS (ESO) in 2000/2001.

could rotate without breaking up and using the value of $\omega$, we obtain $i=35^{\circ}$. These two determinations of the inclination angle are consistent with the low inclination angle $\left(i=30^{\circ} \pm 5\right)$ deduced from the calculations of Poeckert \& Marlborough (1976) from polarimetric measurements.

From $i=35^{\circ}$ and our determination of $v \sin i=$ $179 \mathrm{~km} \mathrm{~s}^{-1}$, we obtain $f_{\text {rot }}=0.66 \mathrm{~cd}^{-1}$. Using $i=32^{\circ}$ and the corresponding radius as determined from the BCD method, which takes into account rapid rotational and evolutionary effects, and using $v \sin i=175 \mathrm{kms}^{-1}$, we find $f_{\text {rot }}=0.79 \mathrm{c} \mathrm{d}^{-1}$. Therefore, we consider $0.73 \mathrm{c} \mathrm{d}^{-1}$ as the best estimate of the stellar rotational frequency. The uncertainty, however, is still large, as shown in Table 1, where the minimum and maximum values of the two determinations are given, taking into account the extremes of all the errors.

\section{History}

\subsection{Long-term variability}

$\omega$ Ori has a long history of variability in Balmer emission lines, oscillating between B and Be phases (HubertDelplace \& Hubert 1979). The long-term increase and decrease of the $\mathrm{H} \alpha$ emission intensity for the last 25 years are seen in Fig. 1. Note that the maximum of $\mathrm{H} \alpha$ 
observed during the early nineties coincides with a maximum in brightness according to the Hipparcos database (Perryman et al. 1997). Hayes \& Guinan (1984) discovered polarization episodes, which were found to be nicely correlated with the presence of continuum flux and color change, weakly correlated with $\mathrm{H} \alpha$ emission strength variations, but not correlated with enhanced wind variability (Sonneborn et al. 1988). The star showed recurrent light bursts every 11 months (long-term period) detected from the ground (Bergin et al. 1989; McDavid et al. 1996) which are compatible with the timescales given by Hipparcos (Hubert \& Floquet 1998).

At the time of the MuSiCoS observations, the star was about to reach a minimum of the long-term photometric period and was close to a minimum of a $\mathrm{Be}$ phase. However, emission was still present in the wings of some lines during the campaign (e.g. $\mathrm{H} \alpha, \mathrm{H} \beta$, He I 5876, 6678, 7065, Si II 6347, C II 6578, 6583). The $\mathrm{H} \alpha$ line had a double-peaked emission $(R \sim V)$ profile with $I_{\max } / I_{\mathrm{c}}=1.4$. Note in Fig. 1 that the emission has risen again since the end of the year 2000 .

\subsection{Short-term variability}

Light modulation has been detected with a period of 1.96 days in optical flux (Balona et al. 1992), whereas the stellar wind seen in the UV lines is modulated by a shorter period: about $1 \mathrm{~d}$ in September 1987 and $1.2 \mathrm{~d}$ in February 1996 (Peters \& Gies 2000). The period derived from a CCF (Cross Correlation Function) method on the photospheric lines from these UV spectra is $1.28 \mathrm{~d}$. These results were interpreted as cyclic variability in the wind of the star, but the phenomenon could be caused by a localized region of mass loss sweeping past the lineof-sight as the star rotates. Recently Balona et al. (2001, hereafter B01) found an averaged period of $0.97 \mathrm{~d}$ using spectroscopic and photometric measurements, similar to the one found by Neiner et al. (2002, see also Henrichs et al. 2000b). B01 could not discriminate between NRPs and rotation as an explanation for this periodicity, but chose to describe it in terms of co-rotating circumstellar clouds.

\section{Observations and data reduction}

\subsection{Observations}

$\omega$ Ori was intensively observed in 1998 from November 23 to December 15 with 8 telescopes (see Table 2 and Fig. 2):

- in France, at the Observatoire de Haute Provence (OHP), with the $1.52 \mathrm{~m}$ telescope equipped with the spectrograph AURELIE $(R=22000, \lambda=6520$ $6720 \AA)$;

- also at the OHP, with the $1.93 \mathrm{~m}$ telescope equipped with the fiber-fed échelle spectrograph ELODIE $(R=$ $42000, \lambda=3900-6800 \AA$ );

- in the USA, at Kitt Peak National Observatory, with the $0.9 \mathrm{~m}$ coudé feed telescope equipped with the
Time Coverage for Omega Orionis

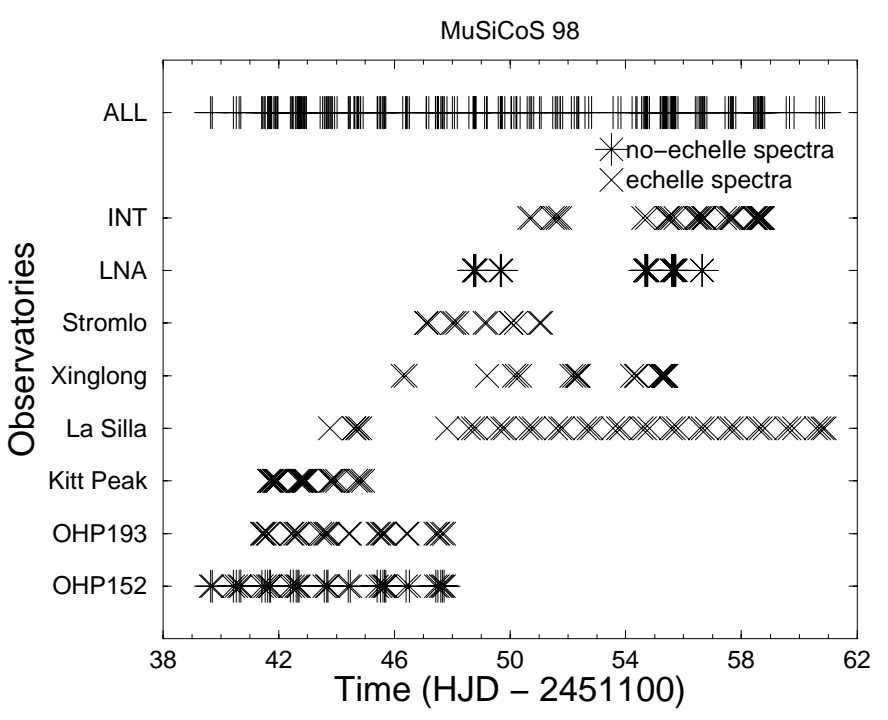

Fig. 2. MuSiCoS 98 observations of $\omega$ Ori with breakdown for each site.

échelle spectrograph $(R=65000, \lambda=4070-5160$ or 5300-7000 А);

- in Chile, at the European Southern Observatory (ESO)/La Silla, with the $0.9 \mathrm{~m}$ Dutch telescope equipped with the Landessternwarte Heidelberg échelle spectrograph HEROS $(R=20000, \lambda=3450$ 5600 and 5800-8650 A simultaneously);

- in China, at the Xinglong Station, with the $2.16 \mathrm{~m}$ telescope equipped with the échelle spectrograph $(R=$ 43000 or $35000, \lambda=3500-7000$ or $5500-8500 \AA$ respectively);

- in Australia, on Mount Stromlo, with the 1.9 m telescope equipped with the échelle spectrograph $(R=$ $35000, \lambda=4800-6800 \AA)$;

- in Brazil, at the Laboratorio Nacional de Astrofisica (MCT/LNA), with the $1.6 \mathrm{~m} \mathrm{B \& C}$ telescope equipped with the coudé spectrograph $(R=60000, \lambda=6563$ or $6678 \AA$ );

- on the Canary Islands, at La Palma, with the $2.5 \mathrm{~m}$ INT telescope equipped with the échelle spectrograph MUSICOS $(R=35000, \lambda=3900-6700 \AA)$.

Bias, flat fields and wavelength calibration exposures produced by Th-Ar lamps were obtained every 2-4 hours. An early-type comparison star with low $v \sin i, \gamma \mathrm{Peg}$, telluric standard stars such as $\alpha$ Peg and velocity standard stars such as $\beta$ Lep were observed from each site to correct for telluric lines and check for possible problems in the calibration.

\subsection{Reduction}

The instruments lack homogeneity: gratings, filters, ranges of wavelengths in the orders of échelle spectra and detectors are different, which hampered the data reduction procedure. The OHP152, Kitt Peak, LNA, 
Table 2. Journal of MuSiCoS 98 observations of $\omega$ Ori.

\begin{tabular}{lllll}
\hline \hline $\begin{array}{l}\text { HJD span(d) Site } \\
(2451100+)\end{array}$ & \multicolumn{2}{l}{$\begin{array}{l}\text { N. of } \\
\text { spectra time (min) }\end{array}$} \\
\hline $39.64-47.72$ & OHP152 & 33 & $15-20$ & $200-500$ \\
$41.42-47.72$ & OHP193 & 29 & $15-20$ & $300-400$ \\
$41.66-44.93$ & Kitt Peak 36 & $15-25$ & $100-200$ \\
$43.78-60.88$ & ESO & 47 & 20 & $100-300$ \\
$46.29-55.41$ & Xinglong & 22 & 20 & $200-300$ \\
$47.10-51.08$ & Stromlo & 13 & 10 & $150-200$ \\
$48.74-56.65$ & LNA & 27 & $18-25$ & $300-500$ \\
$50.66-58.75$ & INT & 36 & $7-30$ & $50-150$ \\
\hline
\end{tabular}

Mount Stromlo and INT data were reduced using IRAF ${ }^{1}$ software. The OHP193, ESO and Xinglong data were reduced with MIDAS $^{2}$ software. A correction for the motion of the Earth was applied and the telluric lines were removed when possible. Finally each line in each individual spectrum has been normalized by selecting precise continuum points around the line, and rebinned on a carefully chosen equidistant wavelength grid $(0.1 \AA)$ using IRAF. The normalization of the spectra is the most critical part of the reduction procedure of multi-site campaigns to obtain homogeneous data (see Hubert et al. 1997).

\section{Line-profile analysis}

Several lines were examined: He I 4471, 4713, 4921, 5876, 6678, C II 4267, Mg II 4481 and Si III 4553. They all show variations. In Fig. 3 we present characteristic examples of the studied line profiles. Note that the red wing of the He I 5876 line is corrupted by an unreliable continuum determination due to a blend of telluric lines. After the mean profile is subtracted, LPVs are very similar for all lines. When emission is present, the variations are seen on a broader domain than $\pm v \sin i$ : Fig. 4 shows the variance of the He I 6678 line, affected by emission, and the unaffected Si III 4553 line. The He I 4713, 4921 and 5876 lines show variations similar to the He I 6678 line, whereas the C II 4267 and Mg II 4481 lines behave similar to the Si III 4553 line. The red wing of the He 4471 line and the blue wing of the Mg II 4481 line contaminate each other.

\subsection{Time series analysis of $L P V s$}

We applied different methods to search for periodicity in the LPVs. The results of the two best methods, the Restricted Local Cleanest (RLC, based on Foster 1995; Foster 1996, developed by Emilio 1997 and

1 IRAF is distributed by the National Optical Astronomy Observatories, which is operated by the Association of Universities for Research in Astronomy (AURA), Inc., under cooperative agreement with the National Science Foundation.

2 MIDAS is distributed by the European Southern Observatory (ESO).
Examples of line variations

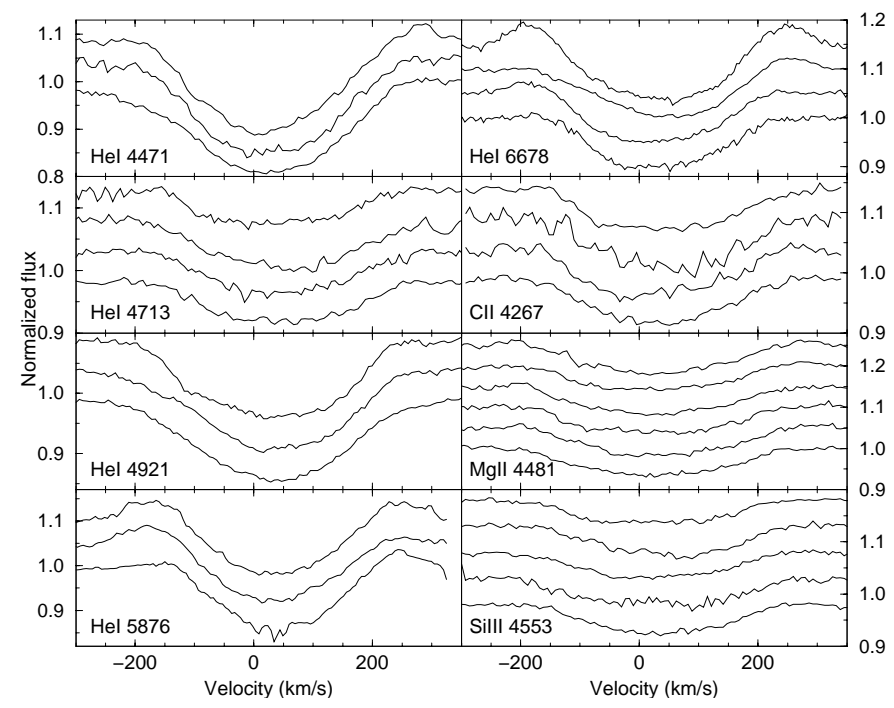

Fig. 3. Examples of typical variations for each studied line. The different normalized profiles have been vertically shifted by 0.05 to facilitate comparison.

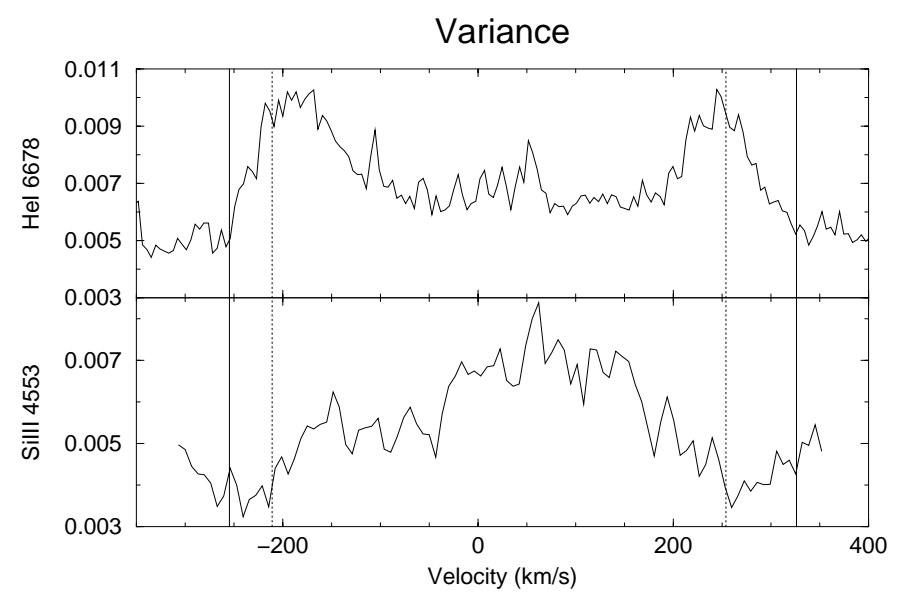

Fig. 4. Variance of the He I 6678 and Si III 4553 lines. The limits of the He I 6678 line are indicated as solid lines and of the Si III 4553 line as dotted lines, on both panels.

Domiciano de Souza Jr. 1999 and applied in Domiciano de Souza et al. 2000) and the Least Squares (LS) methods, are presented here.

We have used the RLC method to search, in each wavelength bin, for 30 frequencies in a predefined range from which it computes all possible models with 4 frequencies. Comparing the power of each of these models, it selects the 7 optimal frequencies, while suppressing the aliases. A Local Cleanest (Foster 1995) is then applied, i.e. the RLC looks for 7 points around each of these 7 frequencies to finetune the final frequencies.

With this method the window aliases are removed. However, in case some aliases may remain, the cleaned spectral window is checked and the remaining frequencies are not considered as real (e.g. Fig. 5). Periods longer than the total duration of the observing run of 22 days 


\section{Window Hel 6678}

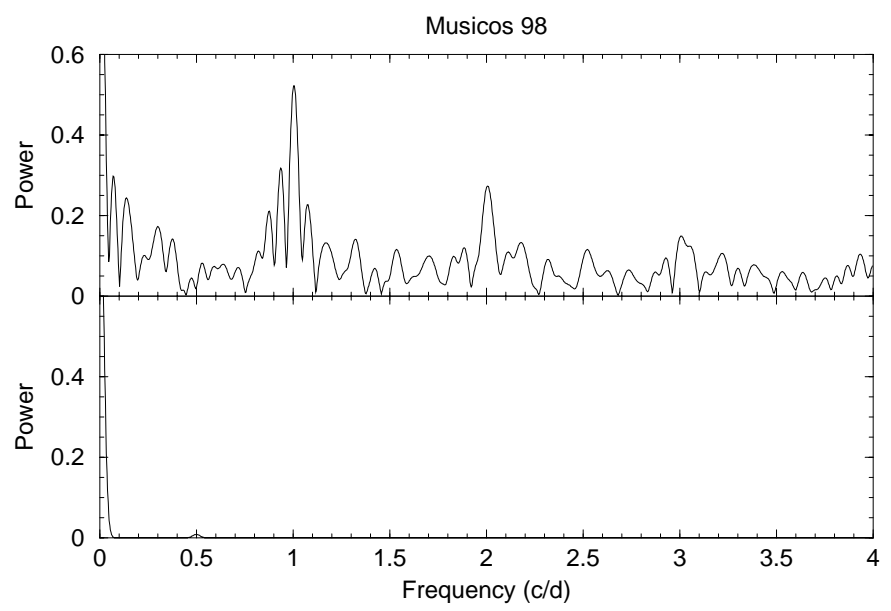

Fig. 5. Top: the spectral window of the MuSiCos 98 campaign for the He 6678 line. Bottom: residual window after applying the RLC method.

$\left(f \leq 0.047 \mathrm{c} \mathrm{d}^{-1}\right)$ cannot be detected. The mathematical frequency resolution of the RLC method is better than $0.01 \mathrm{~cd}^{-1}$, but the accuracy of the results due to the length of the observing run is $0.047 \mathrm{~cd}^{-1}$. We also assigned weights to each spectrum according to the $S / N$ ratio measured in the continuum next to the line, but this did not change the results.

The LS method, as applied by Kambe et al. (1993a), considers the whole line profile at the same time, i.e. all wavelength bins together, to determine which frequencies describe the LPVs in the best way. After the first frequency is found, it is removed from the data (prewhitening). Then the program searches for the next frequency in the residual spectra and the procedure is repeated several times.

The results of the time-series analysis for each line with both methods are recorded in Table 3. The search has been done simultaneously for all sites where data for the specific line were available. This gives strong confidence in the frequencies detected in many lines: they cannot be attributed to window aliasing as the database for each line differs. The spectral window for the He I 6678 line is shown as an example in Fig. 5 (top).

For each line, the greyscale periodogram, mean line profile and power spectrum are shown in Fig. 6. Several frequencies are detected:

(a) a powerful frequency was clearly found at $f_{1}=$ $1.03 \mathrm{c} \mathrm{d}^{-1}\left(P_{1}=0.97 \mathrm{~d}\right)$, identical to the one published by B01;

(b) a second frequency is present at $f_{2}=0.46 \mathrm{~cd}^{-1}$ $\left(P_{2}=2.17 \mathrm{~d}\right)$;

(c) a frequency $f_{3}=0.56 \mathrm{~cd}^{-1}\left(P_{3}=1.78 \mathrm{~d}\right)$ is also detected, together with its first harmonic $1.12 \mathrm{~cd}^{-1}$. This frequency is probably a combination of $f_{1}$ and $f_{2}$ as $f_{1}-f_{2}=0.57 \mathrm{c} \mathrm{d}^{-1}$

(d) a frequency around $f_{4}=0.82 \mathrm{~cd}^{-1}\left(P_{4}=1.22 \mathrm{~d}\right)$ is seen in the stronger lines, similar to the one detected in
Table 3. Main frequencies (in $\mathrm{cd}^{-1}$ ) deduced from the analysis of several lines for all sites for which data was available, indicated in Col. 2, with the number of spectra in Col. 3. In Cols. 4 and 5 the frequencies found by the RLC method are shown in normal fonts while the results of the LS method are shown in boldface. The resolution is $0.01 \mathrm{c} \mathrm{d}^{-1}$ whereas the accuracy of the frequencies is $0.047 \mathrm{~cd}^{-1}$.

\begin{tabular}{lllll}
\hline \hline \multirow{2}{*}{ Line } & \multirow{2}{*}{ Sites } & Spec. & \multicolumn{2}{l}{ Frequencies $\left(\mathrm{c} \mathrm{d}^{-1}\right)$} \\
& & & $f_{1}$ & $f_{2}$ \\
\hline He I 4471 & $3,4,5,8$ & 125 & $\mathbf{1 . 0 4}$ & $0.50 / \mathbf{0 . 5 0}$ \\
He I 4713 & $2,3,4,8$ & 131 & 1.01 & $\mathbf{0 . 4 3}$ \\
He I 4921 & $2,3,4,6,8$ & 130 & $1.03 / \mathbf{1 . 0 3}$ & 0.47 \\
He I 5876 & $2,3,4,5,6,8$ & 147 & $1.03 / \mathbf{1 . 0 4}$ & $0.48 / \mathbf{0 . 4 8}$ \\
He I 6678 & $1,3,4,5,6,7,8$ & 198 & $1.03 / \mathbf{1 . 0 4}$ & $0.46 / \mathbf{0 . 4 6}$ \\
C II 4267 & $2,3,4,8$ & 120 & $1.03 / \mathbf{1 . 0 4}$ & \\
Mg II 4481 & $2,3,4,5,8$ & 125 & $1.04 / \mathbf{1 . 0 3}$ & 0.48 \\
Si III 4553 & $2,3,4,5,8$ & 125 & $1.04 / \mathbf{1 . 0 3}$ & 0.48 \\
\hline
\end{tabular}

Sites: $1=$ OHP152, $2=$ OHP193, $3=$ Kitt Peak, $4=$ ESO, $5=$ Xinglong, $6=$ Mt. Stromlo, $7=\mathrm{MCT} / \mathrm{LNA}, 8=\mathrm{INT}$.

UV data of February 1996 (Peters \& Gies 2000), but the signal is too weak to be studied here. Note that this frequency is close to the rotational frequency determined in Sect. 2.

Finally, a slow variation ( $\sim$ weeks $)$ is seen over the duration of the observing run.

\subsection{Centroid velocity}

For several lines we measured the centroid velocity of the line profiles, which corresponds to the first velocity moment (Balona 1986). All lines show a periodic variation with a frequency of $1.03 \mathrm{c} \mathrm{d}^{-1}$ corresponding to $f_{1}$, shown as a function of phase in Fig. 7, together with a best sine fit. For the C II 4267 line, the high noise level prevented a good fit. The mean centroid velocity $V_{\text {mean }}$, the amplitude of velocity variations $A$ and the phase shift $\Phi$ of the sine fits compared to phase 0 vary for the different lines. Although such differences have already been detected in other Be stars (Štefl et al. 2000), the ones seen here could be due to the limited accuracy of the velocity determinations and the fact that the variations may not be sinusoidal. On average for He I lines, except the He I 4471 line, we obtain $V_{\text {mean }}=34.3 \mathrm{~km} \mathrm{~s}^{-1}, A=3.7 \mathrm{~km} \mathrm{~s}^{-1}$ and $\Phi=0.2$. For purely photospheric lines, except the C II 4267 line, we obtain $V_{\text {mean }}=28.3 \mathrm{~km} \mathrm{~s}^{-1}, A=4.0 \mathrm{~km} \mathrm{~s}^{-1}$ and $\Phi=0.1$. We consider the differences as not significant.

\section{3. $v$ sin $i$ and apparent variations}

The high sampling rate and long duration of the campaign cancel all short-term variations (days) in the mean spectrum of each line. These mean spectra are then not 

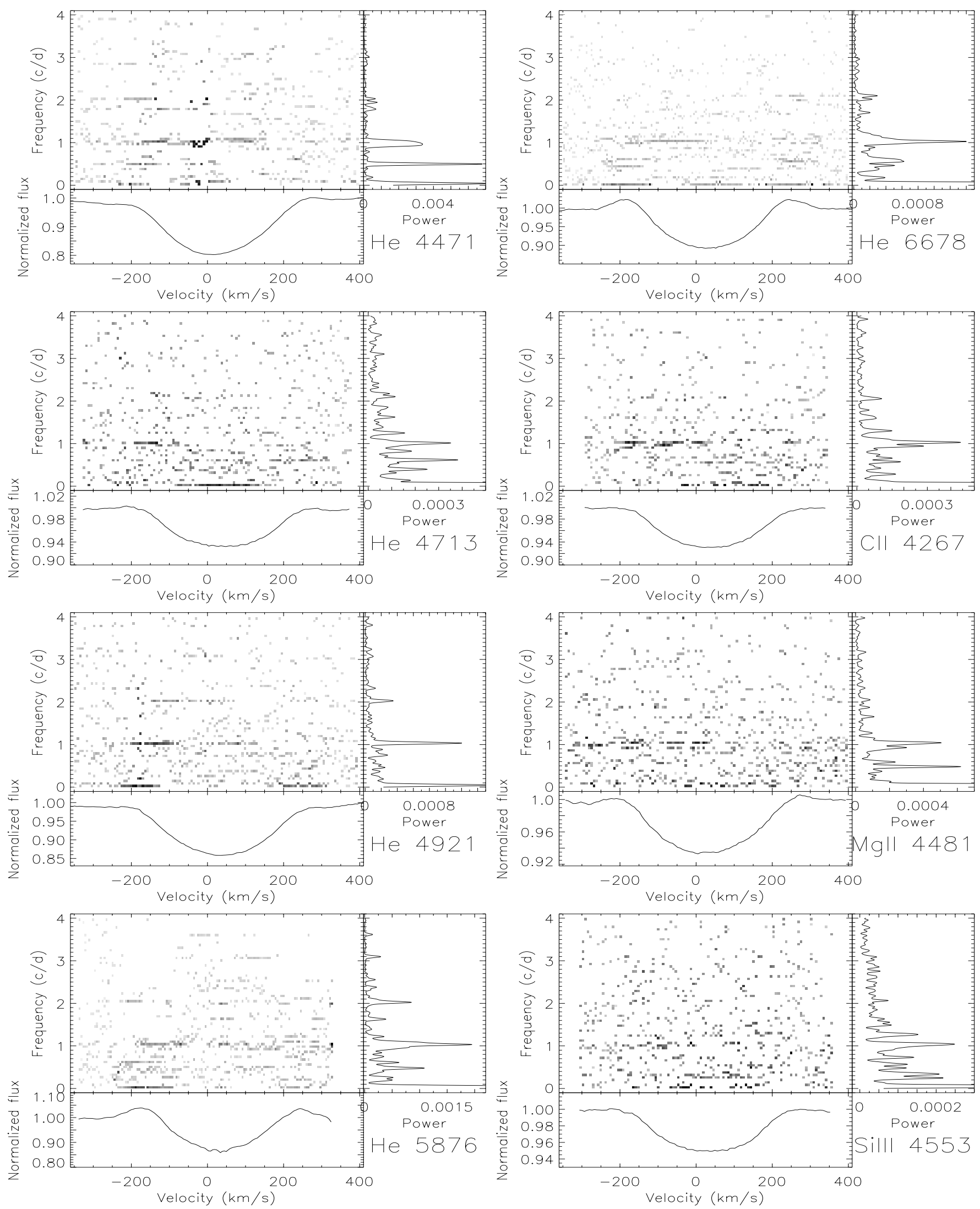

Fig. 6. For each line, the mean line profile is plotted in the bottom panel for the velocity range with a well defined continuum level. The corresponding greyscale periodogram, obtained with the RLC method, is shown in the upperleft panel. The associated power spectrum, smoothed with a gaussian filter and summed over the domain $[-350,400] \mathrm{km} \mathrm{s}^{-1}$ when possible, is shown in the right-hand panel. When the edges of the domain are perturbed by other lines, the sum is taken over the plotted domain (see mean line and greyscale panels). 


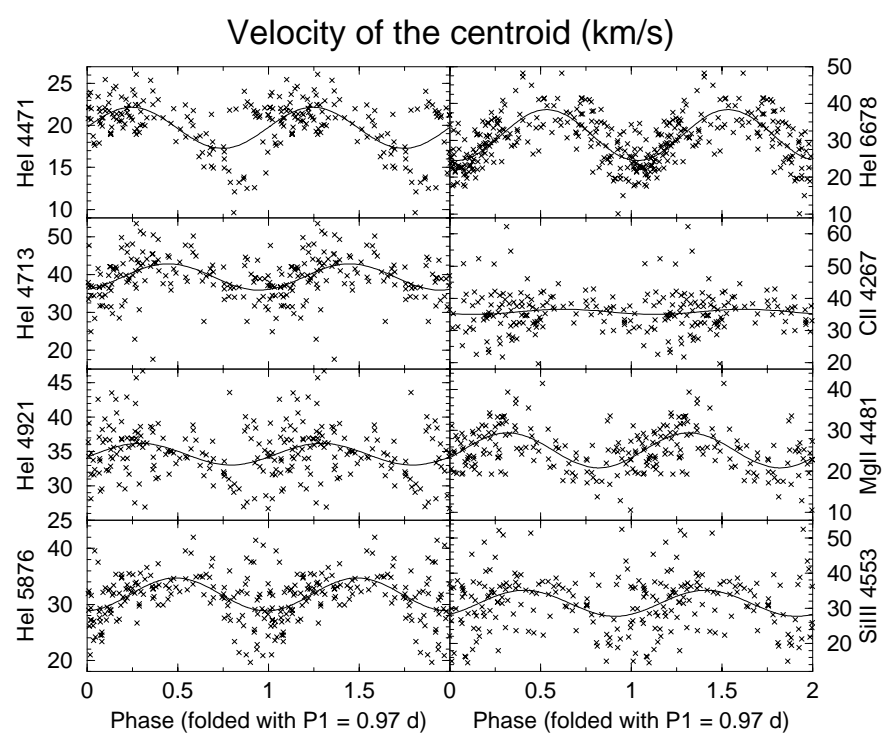

Fig. 7. Centroid velocities (in $\mathrm{km} \mathrm{s}^{-1}$ ) of the different lines folded in phase with frequency $f_{1}=1.03 \mathrm{~cd}^{-1}$.

perturbed by pulsational variations if present, but the mean line width will be different from the one of a similar non-pulsating star because of the pulsational velocity component.

However, $v \sin i$ can be well determined by applying a Fourier transform analysis (Gray 1976) to the mean line profiles of a pulsating star. An additional broadening can introduce new minima in the Fourier transform of the profile, but the determination of $v \sin i$ is unaffected since the position of the first minimum of the rotation profile cannot be modified. Thus, using this method, the estimate of $v \sin i$ is not significantly affected by emission in the line wings (Jankov et al. 2000).

The result for each line (He I 4009, 4026, 4144, 4471, 4713, 4921, 5876, 6678, CII 4267, Mg II 4481 and Si III 4553) is reported in Table 4 and plotted in Fig. 8 for the eight main lines studied in this work. The values are consistent with each other, except for the He I 5876 line, but recall that its red wing is not reliable, and for the He I 6678 line at a lower degree. The $v \sin i$ value of the pulsating star can then be averaged, using all lines in Table 4 except the He I 5876 line, giving $178.8 \pm 3.5 \mathrm{~km} \mathrm{~s}^{-1}$.

B01 determined $v \sin i$ for most of these lines (see Table 4), using calculated intrinsic line profiles. They found similar results for the HeI lines, but a discrepancy between He I $\left(173 \mathrm{~km} \mathrm{~s}^{-1}\right)$ and purely photospheric $\left(226 \mathrm{~km} \mathrm{~s}^{-1}\right)$ lines and proposed to explain this difference as being due to the presence of circumstellar material in the He I lines.

Although the emission intensity during the MuSiCos 98 observations $\left(I_{\max }(\mathrm{H} \alpha)=1.4\right)$ was higher than during their observations $\left(I_{\max }(\mathrm{H} \alpha)=1.1\right)$, this discrepancy is not seen in this work, thanks to the Fourier method, since the position of the first minimum of the rotation profile is not very sensitive to emission in the line wings. From the
Fourier Amplitude (log)

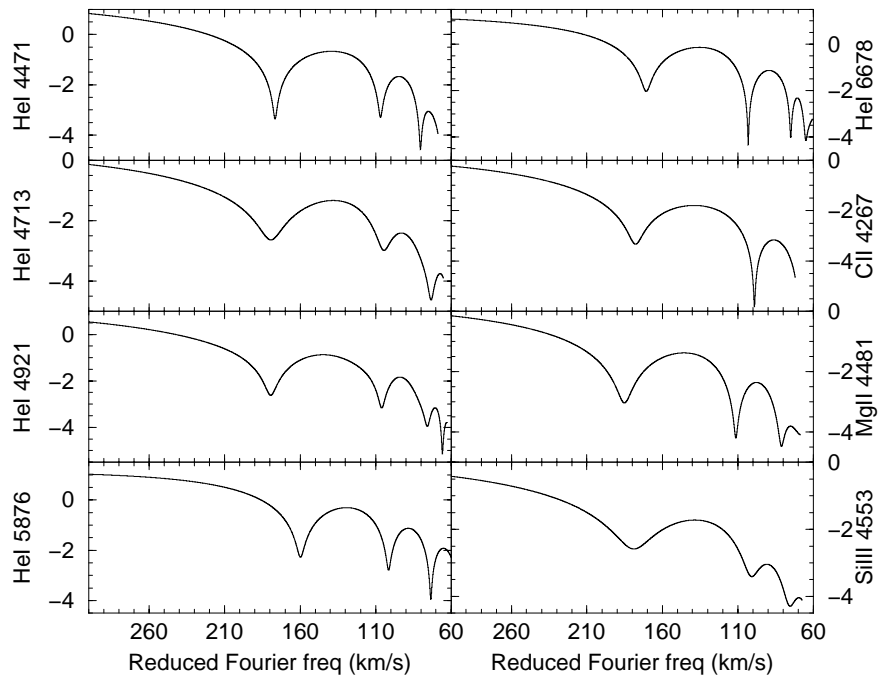

Fig. 8. Amplitude (in logarithmic scale) of the reduced Fourier frequency for each line. The first minimum gives $v \sin i$ in $\mathrm{kms}^{-1}$.

Table 4. Determined values of $v \sin i\left(\mathrm{~km} \mathrm{~s}^{-1}\right)$ for each individual line using the Fourier transform analysis, compared to the values given by B01.

\begin{tabular}{lll}
\hline \hline Line & $\begin{array}{l}v \sin i \\
\text { this paper }\end{array}$ & B01 \\
\hline He I 4009 & 180.8 & \\
He I 4026 & 180.9 & 177 \\
He I 4144 & 179.0 & 173 \\
He I 4471 & 176.6 & 174 \\
He I 4713 & 179.4 & 180 \\
He I 4921 & 179.5 & 183 \\
He I 5876 & $159.8 ?$ & 156 \\
He I 6678 & 170.6 & 173 \\
C II 4267 & 177.7 & 214 \\
Mg II 4481 & 185.1 & 233 \\
Si III 4553 & 178.8 & 223 \\
\hline
\end{tabular}

results shown here, we conclude that $v \sin i$ is of the same order for He I and purely photospheric lines.

Independently of the results shown above, we determined $v \sin i$ using a selection of the 98 available IUE spectra of $\omega$ Ori. We convolved the spectrum of a slowly rotating $\left(v \sin i=40 \mathrm{~km} \mathrm{~s}^{-1}\right)$ reference star of the same spectral type $\left(\pi^{4}\right.$ Ori) with a rotational profile. Using a least-squares method and about 800 lines, the best fit is achieved at $v \sin i=169 \mathrm{~km} \mathrm{~s}^{-1}$. This implies that the best value of $v \sin i$ for $\omega$ Ori is $\sqrt{169^{2}+40^{2}}=174 \mathrm{~km} \mathrm{~s}^{-1}$. This result is in agreement with the one obtained by Fourier analysis, giving strong confidence in the obtained value.

In the rest of this work we adopt the value determined by the Fourier method: $v \sin i=179 \mathrm{~km} \mathrm{~s}^{-1}$. 


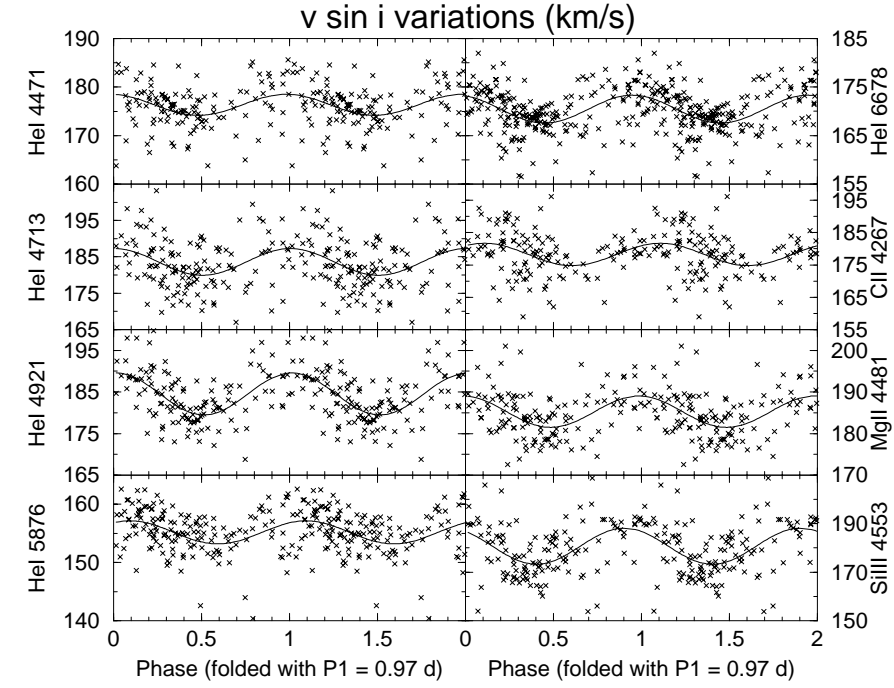

Fig. 9. Apparent $v \sin i\left(\right.$ in $\mathrm{km} \mathrm{s}^{-1}$ ) for each line folded in phase with frequency $f_{1}=1.03 \mathrm{~cd}^{-1}$. A best sinusoidal fit is overplotted.

Inspecting individual spectra of the MuSiCoS campaign, a variation in time is observed in the apparent $v \sin i$ with frequency $1.03 \mathrm{c} \mathrm{d}^{-1}$ corresponding to $f_{1}$. An overplot with a best fit sinusoid is diplayed in Fig. 9. In the frame of NRPs, such variations can be interpreted as a consequence of a horizontal velocity field and/or temperature oscillations.

\subsection{Slow variation $(\sim$ weeks)}

A variation over the length of the run is found in the lineprofile analysis, but is hard to characterize from the LPV as its duration is comparable to the length of the MuSiCoS 98 campaign. However, this variation can clearly be seen from other parameters such as the peak intensities or the equivalent widths of the lines.

\subsubsection{Peak intensities}

All the lines affected by emission $(\mathrm{H} \alpha, \mathrm{H} \beta$, He I 5876, 6678, 7065, Si II 6347, C II 6578, 6583) show the same kind of peak variations. Figure 10 shows an example of the $\mathrm{H} \alpha$ line and the $\mathrm{C}$ II doublet at 6578 and $6583 \AA$ A: blue and red emission peaks are seen. Figure 11 shows the summed Violet + Red emissions of the H $\alpha$, He I 5876, 6678, 7065 and Si II 6347 lines, while Fig. 12 shows the separation in the peaks of the He I 5876, 6678 and $\mathrm{H} \alpha$ lines.

The peak separation of the He I lines fluctuates during the first part of the run, with a daily difference up to $60 \mathrm{~km} \mathrm{~s}^{-1}$ between HJD 2451144.5 and HJD 2451148, but the temporal distribution of data between those days is unequal. Then it progressively decreases again until the end of the observing campaign.
Halpha and Cll doublet

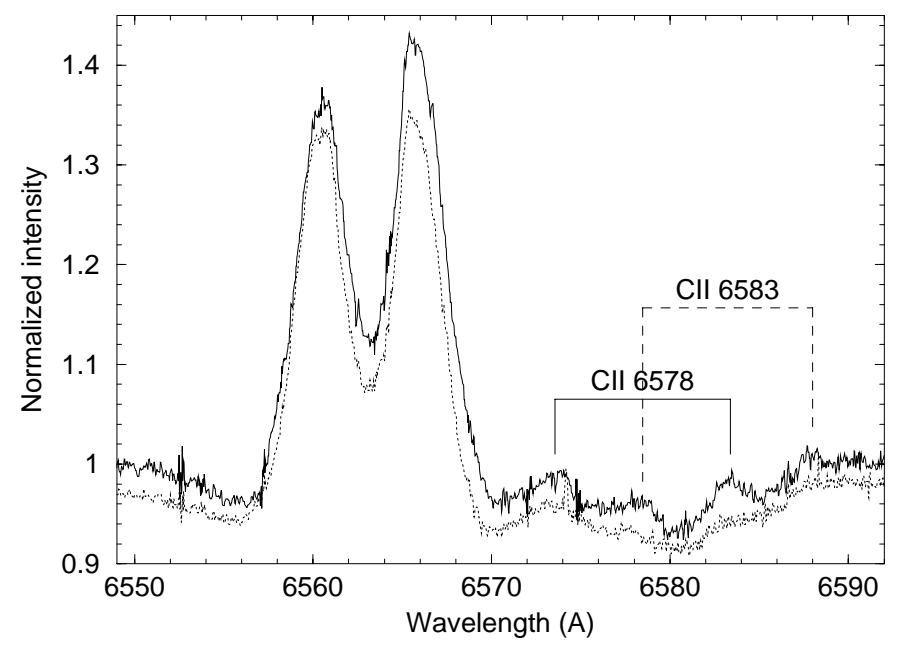

Fig. 10. Example of variable emission peaks in the $\mathrm{C}$ II doublet at 6578 and $6583 \AA$ near the $\mathrm{H} \alpha$ line at HJD 2451142.6 and at HJD 2451147.6 (dotted, and shifted by -0.02 in intensity for clarity). The emission is less strong in the red wing of the $\mathrm{H} \alpha$ and $\mathrm{C}$ II doublet lines.

The peak separation of the $\mathrm{H} \alpha$ line slowly varies over the run; a minimum occurs when the peak separation of the He I lines fluctuates more conspicuously, followed by a gradual increase until HJD 2451155 and finally a slow decrease similar to the He I lines. Nevertheless, a fluctuation at HJD 2451147-48 is still visible.

This evolution is typical for an emission line outburst in a Be star: precursor phase, outburst phase and relaxation phase (see R1), which suggests that an outburst occurred around HJD 2451147. Note that there is no emission in the Si II 6347 line at the beginning of the observing campaign $(V+R=2$, corresponding to the continuum level) and that its maximum in emission does not occur simultaneously with the maxima in emission of the He I lines, but rather precedes them.

The ratio of the Violet and Red emission peaks of all the lines affected by emission also shows strong variability during the first half of the campaign and becomes more stable during the second half. The results for the He I 5876 , 6678, 7065 and C II 6578, 6583 lines are shown in Fig. 13. The similarity between the C II 6578 line and the He I lines is clear. For the C II 6583 line, the variations look different, likely due to the difficulty of the determination of $R$ for this line as this critically depends on the placement of the continuum.

\subsubsection{Equivalent widths}

The variation over the run is also reflected by the change in equivalent width of the red He I lines. Because the variation at the center is in antiphase with respect to the wings of the lines, its effect is cancelled when looking at 
$\mathrm{V}+\mathrm{R}$ intensities

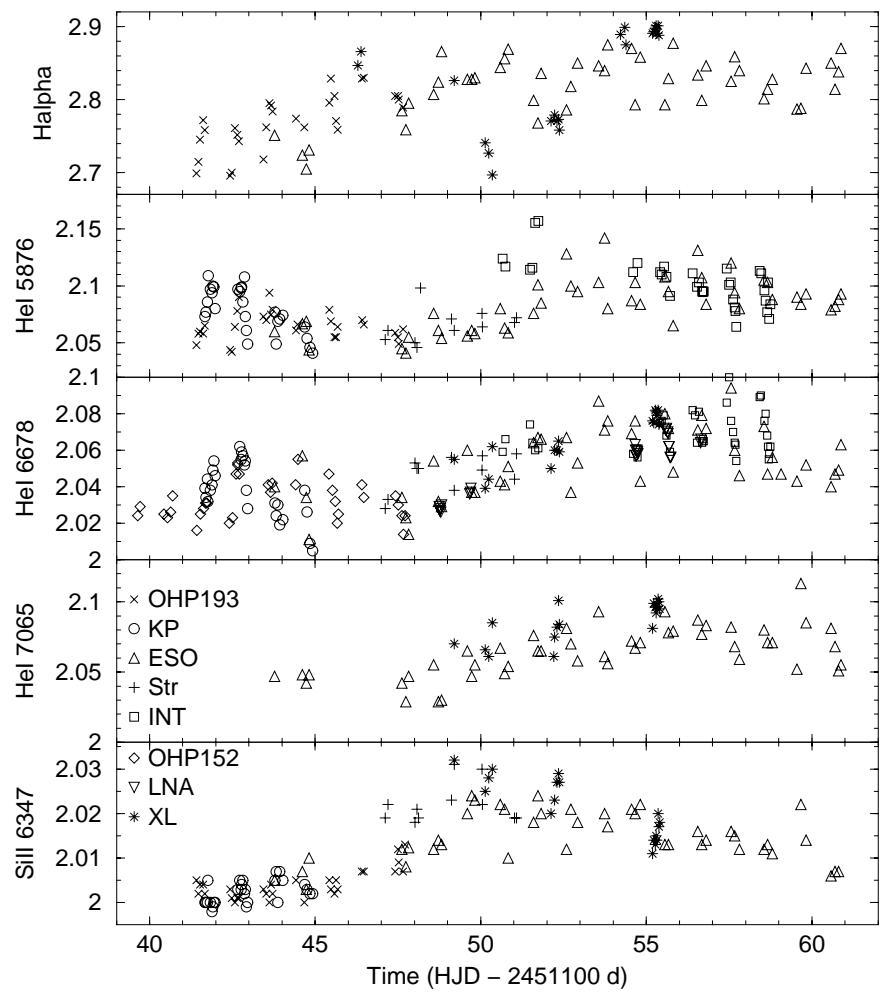

Fig. 11. Variations of the summed Violet + Red emission peaks of the $\mathrm{H} \alpha$, He I 5876, 6678, 7065 and Si II 6347 lines during the MuSiCoS 98 campaign.

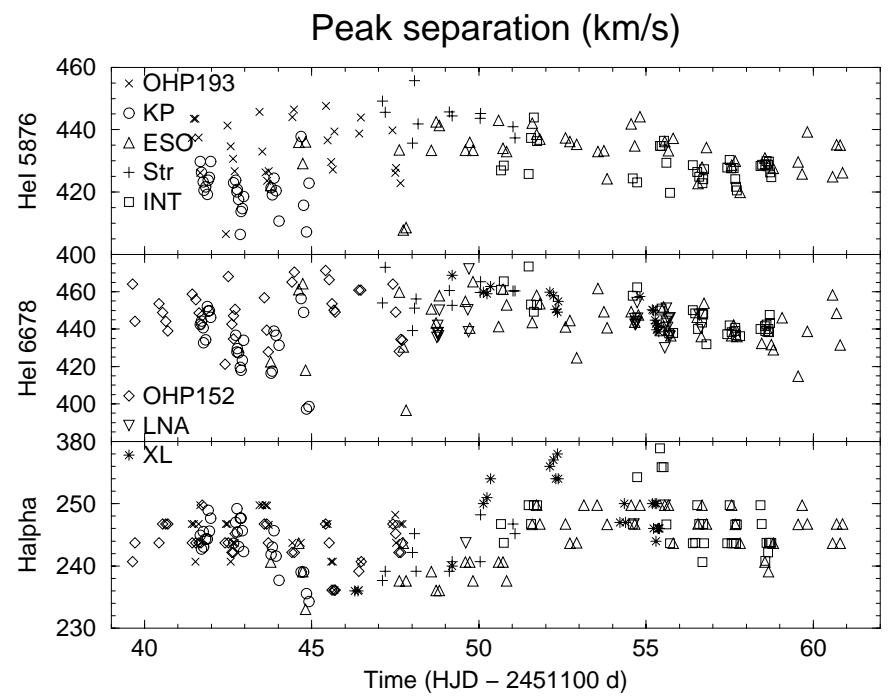

Fig. 12. Variations in $\mathrm{km} \mathrm{s}^{-1}$ of the emission peak separation of the He I 5876, 6678 and $\mathrm{H} \alpha$ lines during the MuSiCoS 98 campaign.

the equivalent width of the whole line. However, studying the wings (blue: $[-350,-100] \mathrm{km} \mathrm{s}^{-1}$ and red: $[180$, $\left.400] \mathrm{kms}^{-1}\right)$ and the center $\left([-100,180] \mathrm{km} \mathrm{s}^{-1}\right)$ of the line separately allows to recover the variation, as shown in Fig. 14.

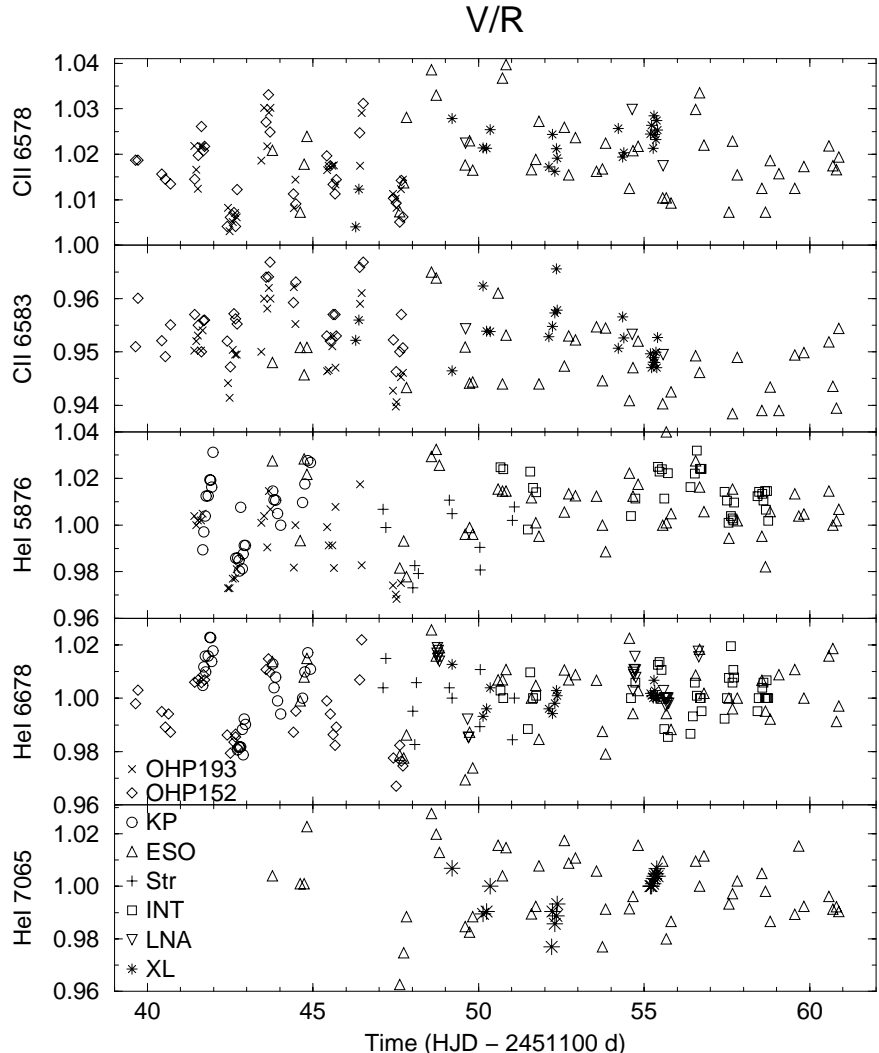

Fig. 13. Variations of the ratio of the Violet over Red emission peaks of the C II doublet at 6578 and $6583 \AA$ and the He I 5876, 6678 and 7065 lines during the MuSiCoS 98 campaign.

\section{Equivalent widths (A) Hel 6678}

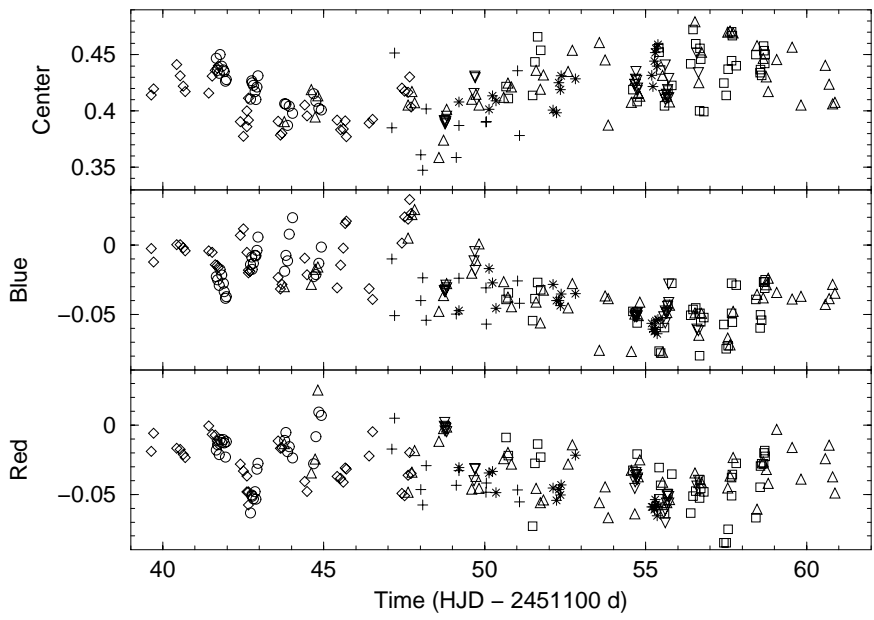

Fig. 14. Equivalent widths variations (in $\AA$ ) of the center and the blue and red wings of the He I 6678 line during the MuSiCoS 98 campaign. See symbol caption in Fig. 12.

We also studied the $\mathrm{H} \delta$ line but no significant changes indicative of an outburst have been detected. A weak tendency of narrower wings and less deep line core is observed between HJD 2451148 and 52. Note, however, that there is almost no emission in $\mathrm{H} \delta$ during the campaign. 


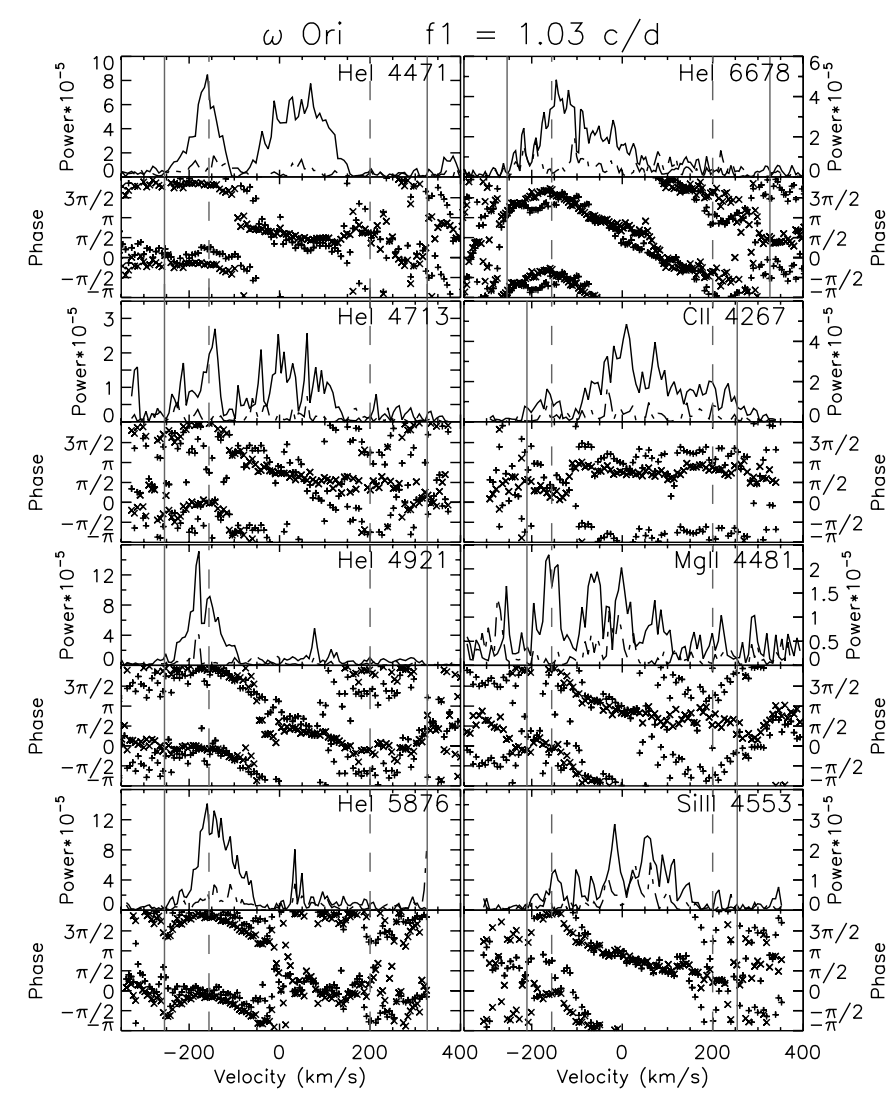

Fig. 15. Power (solid line) and phase (cross symbols) for the frequency $f_{1}=1.03 \mathrm{~cd}^{-1}$ and for its first harmonic at $2.06 \mathrm{~cd}^{-1}$ (power as dashed line and phase as plus symbols). Vertical dotted lines show the limits of the domain determined from the variance; vertical dashed lines show the domain $\pm v \sin i$.

\section{The frequency $f_{1}=1.03 \mathrm{~cd}^{-1}$}

The observed variations can be interpreted in terms of a model with corotating spots or clouds (B01) or with the presence of a NRP mode. In this section we investigate whether the observed frequency $f_{1}$ could be interpreted in the frame of NRPs.

The frequency $f_{1}$ has also been detected in photometry (e.g. B01). From $f_{\text {rot }}$ determined in Sect. 2, we obtain $f_{1} / f_{\text {rot }}=1.4$. This is in favor of the pulsation model as Zorec et al. (2002) showed that $f_{\text {photometry }}$ can hardly represent $f_{\text {rot }}$.

\subsection{Phase diagrams}

For each studied line the phase and power diagrams for the frequency $f_{1}$ are shown in Fig. 15. The $\pm v \sin i$ domain and the velocity domain derived from the variance (see Sect. 5) are shown. The phases were recomputed with a LS technique, as cleaning methods such as RLC give less accurate phase values. Greyscale dynamic spectra as a function of phase are presented in Fig. 16, showing ab-
Table 5. NRP parameters (pulsation degree $l$ and azimuthal order $m$ ) for the frequency $f_{1}=1.03 \mathrm{~cd}^{-1}$ determined from different methods. Cols. 2-3: phase slope determination; Cols. 4-5: same with T\&S correction coefficients; Cols. 6-7: FDI determination.

\begin{tabular}{lllllll}
\hline \hline & \multicolumn{2}{l}{ Phase } & \multicolumn{2}{l}{ T\&S } & \multicolumn{2}{l}{ FDI } \\
Line & $l$ & $|m|$ & $l$ & $|m|$ & $l$ & $|m|$ \\
\hline He I 4471 & $1-2$ & 1 & 2 & $0-1$ & 2 & \\
He I 4713 & 2 & 1 & 2 & $0-1$ & 3 & \\
He I 4921 & 2 & $1-2$ & $2-3$ & 1 & 3 & \\
He I 5876 & 2 & $1-2$ & $2-3$ & 1 & 3 & \\
He I 6678 & 2 & $1-2$ & 3 & 1 & 3 & $2-3$ \\
C II 4267 & $1 ?$ & 1 & $1 ?$ & $0-1$ & 3 & \\
Mg II 4481 & 1 & 1 & 2 & $0-1$ & 3 & \\
Si III 4553 & 2 & $1-2$ & 2 & 1 & 3 & \\
\hline
\end{tabular}

sorption and emission features travelling across the line profiles. These features are clearly seen on the blue side of all lines. They can also be seen travelling back on the red side of strong lines such as He I 6678. This can be explained with the low inclination angle $i$ of the star, so that we view the pulsations on the far side of the star, similar to what has been observed in other Be stars (see $v$ Cyg in Floquet et al. 2000b, 48 Per in Hubert et al. 1997, $\mu$ Cen in Rivinius et al. 1998b).

Note that both the He I and the purely photospheric lines of other species (C II 4267, Mg II 4481 and Si III 4553) show pulsations, which are all in phase with each other. The slope of the phase variation is the same for each line, except for C II 4267, but this line is the weakest one and its phase is less well defined. Once again, note that the power of the pulsations is generally higher at the blue side of the line, especially in the He I lines which show stronger emission. This asymmetry, which has also been observed in other Be stars (see EW Lac in Floquet et al. 2000a, $\mu$ Cen in Rivinius et al. 2001), is especially strong in $\omega$ Ori but remains unexplained. Townsend (2000) suggested that trans-photospheric wave leakage may play a role.

\subsection{Mode determination of the NRPs}

The frequency $f_{1}=1.03 \mathrm{~cd}^{-1}$ found by the time-series analysis can be associated with NRP modes. The slope of the phase diagram gives an estimate of the pulsation degree $l$. The slope of the phase diagram of the first harmonic provides an estimate of the azimuthal order $|m|$ (Fig. 15). For numerous model fits Telting \& Schrijvers (1997, hereafter T\&S) derived that for $l-|m|<2$ the following corrections apply:

$l=0.076+1.110 * \frac{\left|\Delta \Phi_{0}\right|}{\pi}$ 


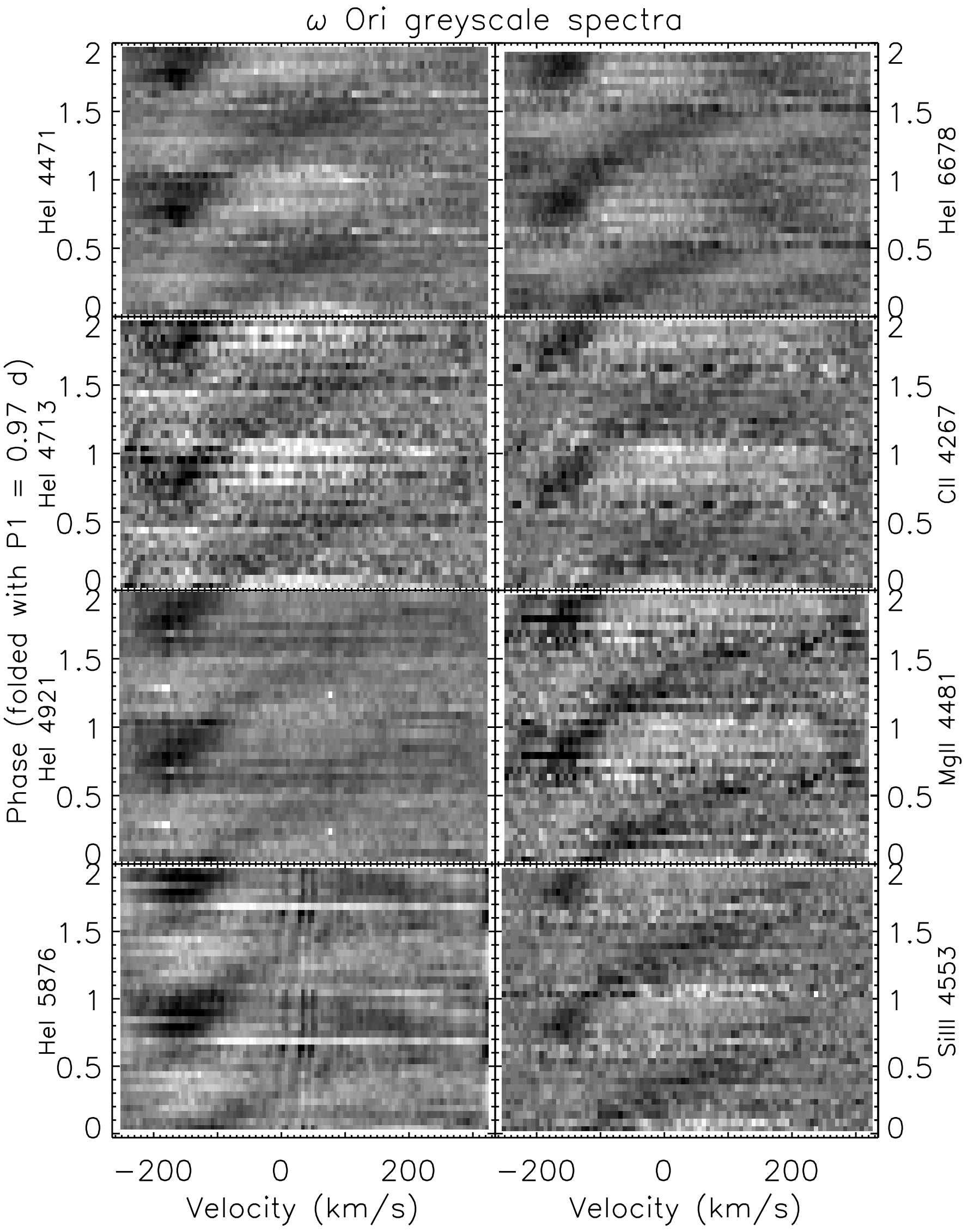

Fig. 16. The greyscale plots of all spectra for each studied line normalized by its mean profile folded in phase with the frequency $f_{1}=1.03 \mathrm{~cd}^{-1}$. The velocity range corresponds to the one determined by the variance of He I lines. 
$|m|=-1.028+0.613 * \frac{\left|\Delta \Phi_{1}\right|}{\pi}$.

The obtained values, however, still have uncertainties of \pm 1 for $l$ and \pm 2 for $|m|$. The results, obtained with and without these correction coefficients, are shown in Table 5.

The mode parameters of the pulsations have also been determined by Fourier Doppler Imaging (FDI, see Kennelly et al. 1992, 1996). In a rapidly rotating star, the pulsation velocity field and the temperature perturbations are mapped onto a wavelength position corresponding to the rotationally induced Doppler shift. When the oscillations are confined to the equatorial region, the obtained normalized wavelength frequency corresponds to $|m|$, otherwise it represents $l$. The FDI method is based on the number of travelling bumps and therefore the mode with $|m|=0$ cannot be detected.

The results are shown in Fig. 17 for each line where the slow trend has been removed. The mode parameters are reported in Table 5 . Note that when the $l$ value was between 2 integer values, it has been averaged to the lowest integer, as our computational tests showed that, for a single mode and adopted stellar inclination, the FDI technique tends to increase the value of the mode parameters.

The frequency $f_{1}$ is then attributed to NRPs with $l=2$ or 3 and $|m|=1,2$ or 3 . However, the pattern of pulsations travelling across the lines seen in Fig. 16 excludes the value $|m|=1$. From a more detailed modeling of $\omega$ Ori (Neiner et al. in preparation), preliminary results show that $|m|=2$ is the most likely case for this star. Therefore we consider in the following the modes $l=2$ or 3 and $|m|=2$.

\subsection{Comparison with theoretical models}

The NRP frequency measured in an inertial frame can be written as (Ledoux 1951):

$\pm f_{\text {puls }}=f_{0} \pm\left(1-C_{n l}\right)|m| f_{\text {rot }}$,

where $f_{\text {puls }}$ is the pulsational frequency in the frame of the observer, $f_{0}$ is the frequency in the stellar frame for a nonrotating star, $f_{\text {rot }}$ is the rotational frequency of the star and the signs are positive (negative) for prograde (retrograde) modes.

For high-order $p$ modes, the higher effects of rapid rotation can be neglected (Dziembowski, private communication) and Eq. (3) transforms to:

$\pm f_{\mathrm{puls}}=\frac{\sqrt{3 G M}}{2 \pi \sqrt{R^{3}}} \sigma_{0} \pm|m| f_{\mathrm{rot}}$.

The value of the positive non-dimensional frequency in the stellar frame

$\sigma_{0}=\frac{2 \pi f_{0}}{\sqrt{4 \pi G<\rho>}}=\frac{2 \pi \sqrt{R^{3}}}{\sqrt{3 G M}} f_{0}$

has been determined by Balona \& Dziembowski (1999, hereafter $\mathrm{B} \& \mathrm{D}$ ), for various values of $l$ for $g$ and $p$ prograde

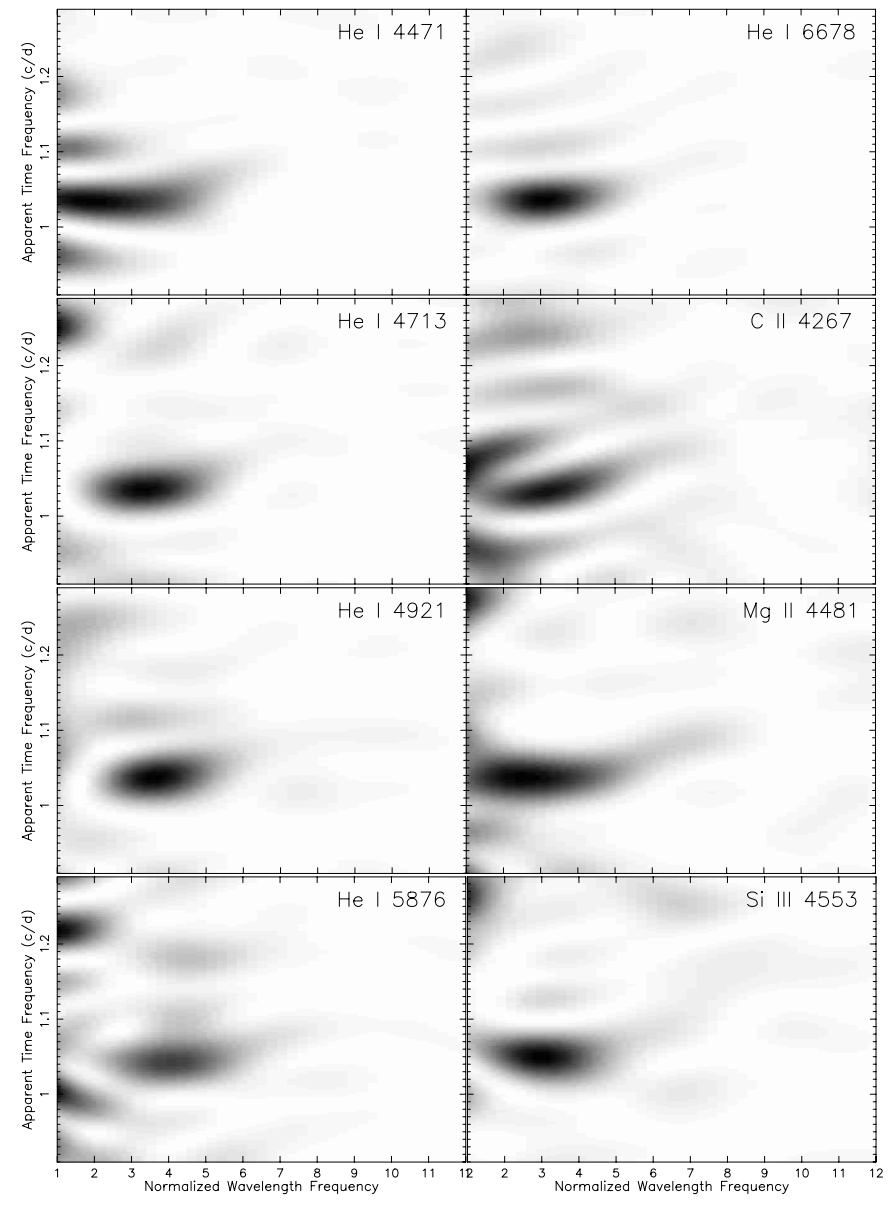

Fig. 17. Greyscale Fourier Doppler Imaging (FDI) results for each studied line. The ordinates provide the time frequency $\left(f_{1}\right)$ while the absisses provide the wavelength frequency, which is an estimate of either the pulsation degree $l$ or the azimuthal order $|m|$. The possible solutions are within an ellipsoidal zone (shown in black).

modes depending on the effective temperature and luminosity of the star. From their calculations for stars with a luminosity between 0.5 and 1.0 mag above the ZAMS, such as $\omega$ Ori, we derive, for prograde $p$ modes, $\sigma_{0} \sim 1.60 \pm 0.4$. This value is also valid in first approximation for retrograde $p$ modes (Dziembowski, private communication).

Taking $f_{\text {puls }}=1.03 \mathrm{~cd}^{-1}, l$ and $|m|$ determined in Sect. 6.2, $M, R$ and $f_{\text {rot }}$ determined in Sect. 2 , the value of $\sigma_{0}$ derived from Eq. (4) is negative for prograde $p$ modes, which is impossible by definition. If $\omega$ Ori hosts a retrograde $p$ mode, we obtain $\sigma_{0}=0.20 \pm 0.04$. This value is incompatible with the one found by B\&D.

Taking slow rotation into account (e.g. Coriolis forces) but no higher effect due to rapid rotation (e.g. departure from sphericity), for high radial order $g$ modes, Eq. (3) transforms to:

$\pm f_{\text {puls }}=\frac{\sqrt{3 G M}}{2 \pi \sqrt{R^{3}}} \sigma_{0} \pm\left(1-\frac{1}{l(l+1)}\right)|m| f_{\text {rot }}$.

If the star hosts a $g$ mode, $\sigma_{0}$ derived from Eq. (6) is negative for a prograde mode, which is impossible by definition, 


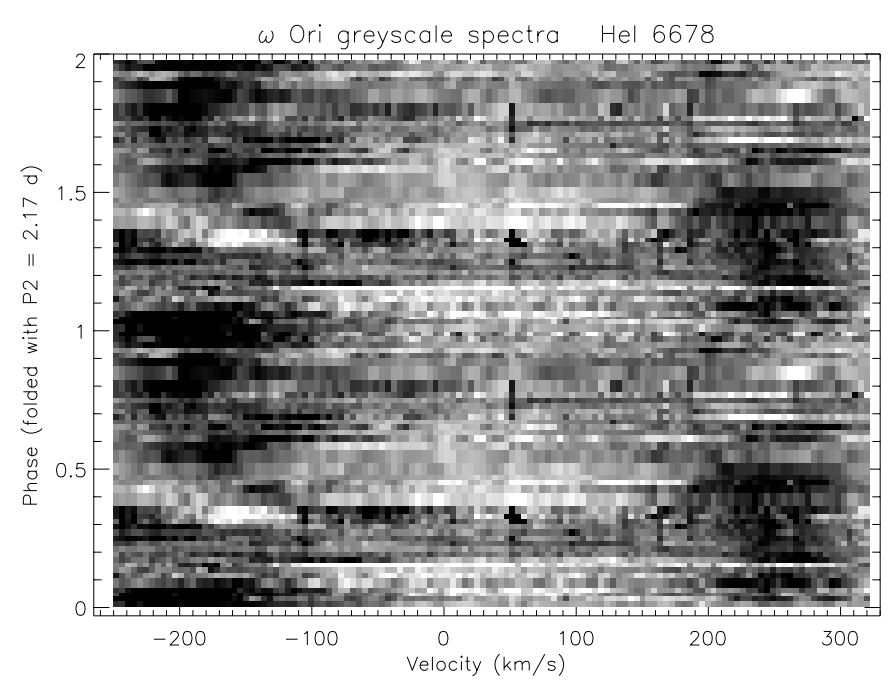

Fig. 18. Greyscale plots of all spectra taken before HJD 2451150 for the He I 6678 lines, normalized by its mean profile, folded in phase using the frequency $f_{2}=0.46 \mathrm{~cd}^{-1}$. The velocity range corresponds to the one determined by the variance in Sect. 5 .

but note that the results are only marginally below zero. $\sigma_{0}=0.08 \pm 0.04$ for $l=2$ and $\sigma_{0}=0.14 \pm 0.04$ for $l=3$ for a retrograde mode. From B\&D, we derive $\sigma_{0} \sim 0.2 \pm 0.05$ for prograde $g$ modes with $l=2$ and $\sigma_{0} \sim 0.35 \pm 0.05$ for prograde $g$ modes with $l=3$. However the approximation of slow-rotation used here may not apply to this star, and the fact that B\&D values are only calculated for prograde modes and are also used here for retrograde modes, may explain the small difference obtained for a retrograde $g$ mode, but also prevent to rule out prograde $g$ modes.

In conclusion, $\omega$ Ori cannot host a $p$ mode with the determined parameters but is likely to host a $g$ mode, possibly retrograde. Spectral modeling is necessary to confirm this result, which is the subject of a follow-up paper.

\section{The frequency $f_{2}=0.46 \mathrm{~cd}^{-1}$}

In spite of the perturbation caused by the frequency $f_{1}$ (travelling patterns on a timescale of about half the period), the frequency $f_{2}$ can be distinguished in the strong He I lines displaying emission in their outer wings such as He I 5876 and 6678 (Fig. 18). It is also suspected in other He I lines weakly affected by emission such as He I 4713 and 4921 , but does not appear in the purely photospheric lines.

Because the frequency $f_{2}$ is weak, the phase diagrams are not as clear, but it seems that the phase is constant. However, the power is strong in the emission wings of the He I lines and the phase is coherent in these regions. Because $f_{2}$ does not appear in the purely photospheric lines but rather in the emission wings of the He I lines, $f_{2}$ is more likely to be associated with orbiting material.

Looking at the $V / R$ data as a function of time (Fig. 13), we see that the frequency $f_{2}$ is not always as

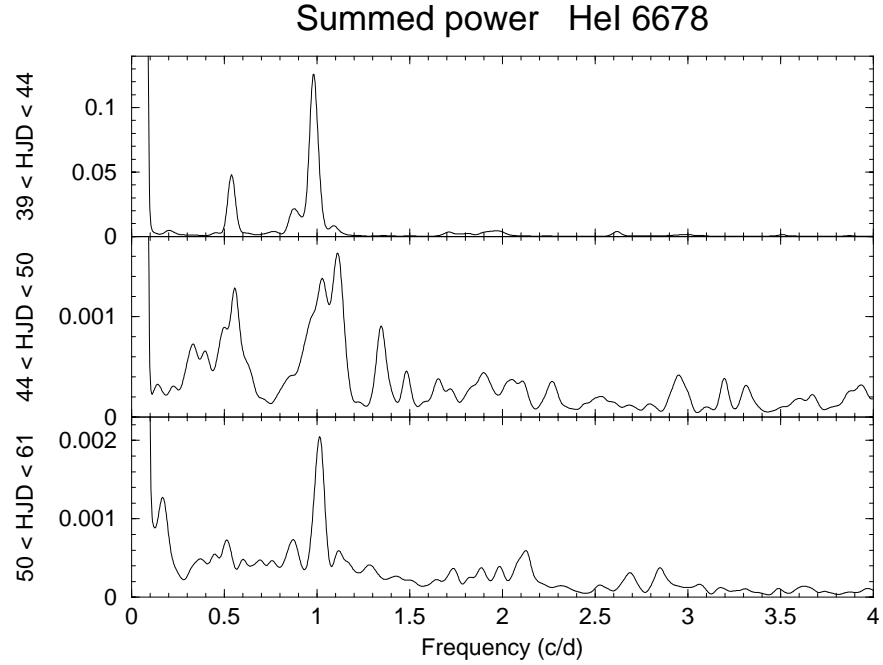

Fig. 19. Summed power obtained with RLC method for the He I 6678 line for 3 datasets: HJD between 2451139 and 2451144, 2451144 and 2451150,2451150 and 2451161. The peaks are smoothed by a Gaussian filter. The accuracy of the frequency determinations for these datasets (between 0.1 and $0.2 \mathrm{c} \mathrm{d}^{-1}$ ) is less good than for the whole MuSiCoS 98 dataset.

strong: it is very strong during the first week of the campaign, then it suddenly fades on a timescale of 2 days between HJD 2451148 and 2451150. A summed power spectrum of the He I 6678 line for three sets of data (HJD between 2451139 and 2451144, 2451144 and 2451150, 2451150 and 2451161) is shown in Fig. 19. As the datasets are smaller, the accuracy of the frequencies is less good, but one can see that the frequency close to $0.5 \mathrm{c} \mathrm{d}^{-1}$ disappears in the last dataset. This suggests that a cloud of material ejected in an outburst orbits around the star during a few periods. Transient periods with similar explanations have been observed in other Be stars (Štefl et al. 2000).

In Fig. 20, we see for the He I 5876, 6678 and 7065 lines that the $V / R$ ratio varies in phase with the frequency $f_{2}$. Dividing the data into 2 parts: the spectra before and after HJD 2451150, clearly shows the difference in strength for this frequency. As an example the He I 6678 line is shown in Fig. 21 with best sine fits. Looking at the ESO data (triangles) in particular assures that this is not due to site-to-site effects.

\section{Discussion}

\section{1. $f_{1}:$ NRPs versus rotation models}

The frequency $f_{1}$ is strong and clearly detected in many line quantities and LPVs. It cannot be due to a one-day window alias. Although the multi-site campaign does not completely remove possible effects of a one-day alias, the RLC method does and its resolution allows to separate $P_{1}=0.97 \mathrm{~d}$ from $1 \mathrm{~d}$ (see Sect. 5.1). Moreover the sinusoidal shape of line quantities folded with $P_{1}$ (Figs. 7 and 9) and the travelling pattern on the greyscale plot 


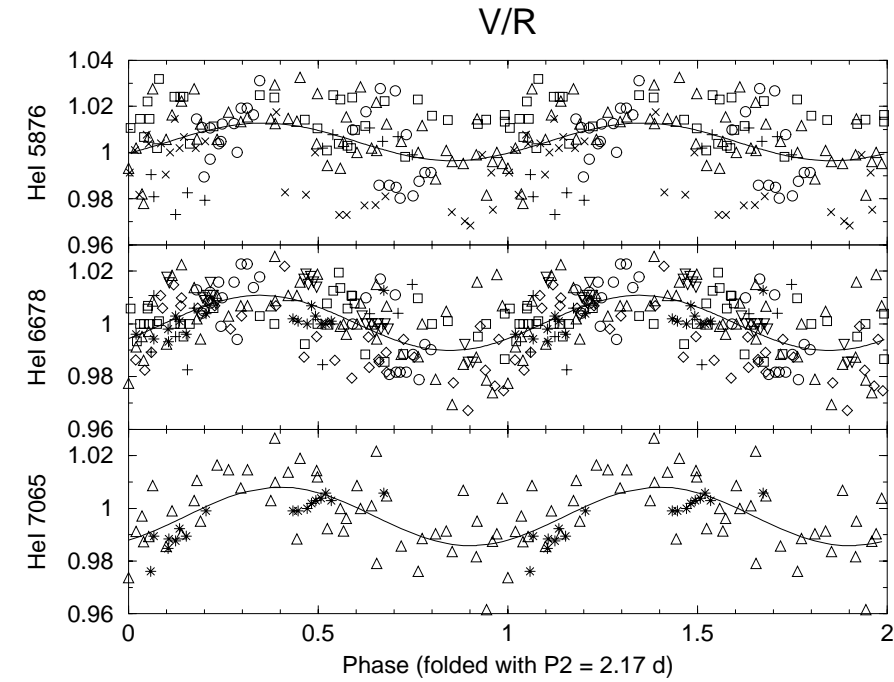

Fig. 20. Ratio of the Violet over Red emission peaks of the He I 5876, 6678 and 7065 lines, showing emission wings, folded in phase with the frequency $f_{2}=0.46 \mathrm{~cd}^{-1}$. Best sine fits are overplotted. See symbol caption in Fig. 12.

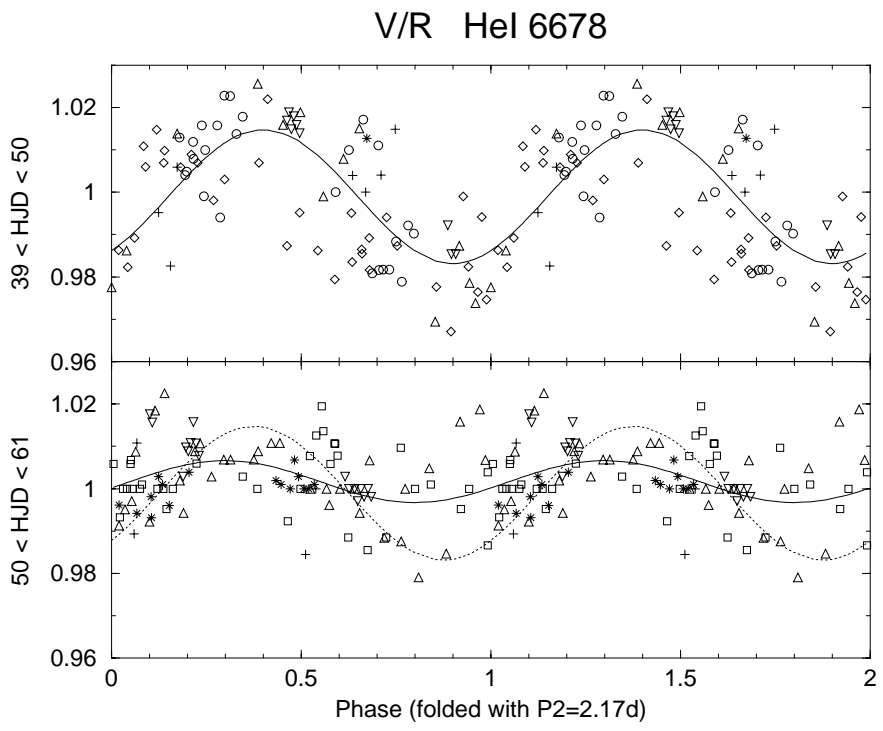

Fig. 21. Ratio of the Violet over Red emission peaks of the He I 6678 line folded in phase with the frequency $f_{2}=0.46 \mathrm{~cd}^{-1}$, before and after HJD 2451150. Best fit sinusoids are overplotted as solid lines. The best-fit curve from the top panel is also reproduced as a dotted line in the lower panel. See symbol caption in Fig. 12.

(Fig. 16) remove any doubt about the reality of this periodicity. It can be attributed to a NRP mode with $l=2$ or $3,|m|=2$ (see Sects. 6.2 and 6.3).

A rotation model with 2 starspots placed exactly opposite each other at the equator will reproduce variations similar to a $l=2,|m|=2 \mathrm{NRP}$ mode, whereas at high latitude it will reproduce variations similar to a $l=3$, $|m|=2 \mathrm{NRP}$ mode. Although this configuration could happen by chance, these kinds of variations have been seen in many Be stars and it is statistically rather unlikely that all these stars have 2 opposite spots exactly at the right latitude. A magnetic dipole with the magnetic axis perpendicular to the axis of rotation could explain two opposite spots at the equator. Nevertheless, no magnetic dipole configuration could explain the other positions of spots, and up to now only one Be star is known to host a magnetic field ( $\beta$ Cep, Henrichs et al. 2000a).

On the other hand, the patterns are also seen travelling back (Fig. 16). This can be explained by NRPs or with starspots, but in the latter case only spots at high latitude will produce a backward moving pattern as strong as the forward moving pattern, as observed here. The pattern created with such spots would then be observed over a smaller velocity range than $\pm v \sin i$, which is obviously not the case.

Therefore, it is more probable that NRPs are involved in $\omega$ Ori. If NRPs are indeed present, the outburst which occurs in $\omega$ Ori could be the result of a beating effect of NRP modes as Rivinius et al. (1998c) showed for the star $\mu$ Cen.

By comparing the photospheric lines and the lines with additional outer emission components, one can investigate the link between the photosphere and the inner circumstellar layers close to the central star.

From the velocity range determined with the variance (Sect. 5) for the He I and other species lines, we know that the extremes of the wings of the strong HeI lines are formed out of the photosphere. These parts of the He I lines also pulsate with the frequency $f_{1}$ (e.g. Fig. 16), as can be seen on a zoom of greyscale spectra, not corrected from the mean spectrum, of the He I 6678 blue wing (Fig. 22). The results are similar for the red wing.

Kambe et al. (1993b) observed the same phenomenon in the Be star $\lambda$ Eri and suggested that it is due to lpv seen in a rotationally accelerated equatorial region. However in $\lambda$ Eri the velocity measured for the outer regions of the lines corresponds to the break-up velocity, which is not the case in $\omega$ Ori. Moreover, the velocity phase in the outer regions of the lines is different from the one in their core (e.g. He I 6678 in Fig. 15).

Therefore, we cannot exclude that some of the ejected material pulsates with the same frequency as the star but with a different velocity phase, probably due to the difference in density between the photosphere and the envelope, and we conclude that a part of the ejected material could still be linked to the star.

\section{2. $f_{2}$ : orbiting material}

The frequency $f_{2}$ is mostly detected in red He I lines affected by emission, but this segregation could be due to the faintness of the other studied lines. Nevertheless, as it is especially powerful in the emission wings of the red He I lines during the first half of the campaign (Fig. 19), this frequency is thought to be due to a cloud of orbiting material. The variations in $V / R$ during the first week of 


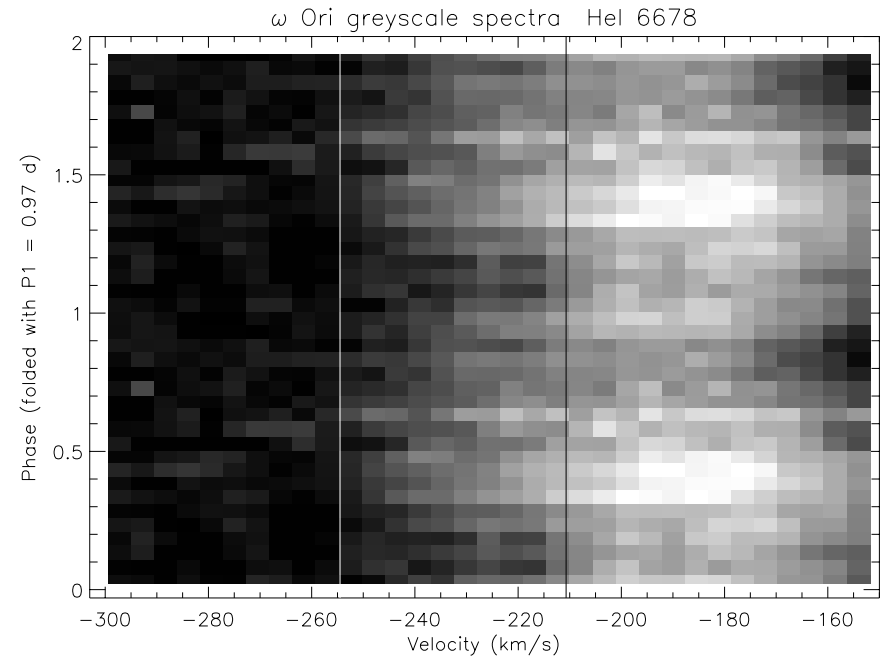

Fig. 22. Greyscale spectra of the blue wing of the He I 6678 line. The black line indicates the velocity region determined from the variance of the purely photospheric lines, whereas the white line indicates the region determined for the He I lines with a circumstellar component.

observations with a period $P_{2}=2.17 \mathrm{~d}$ (Fig. 21) are very similar to the ones observed by several authors in Be stars (e.g. Baade 1982; Smith 1989).

It is known (Hanuschik et al. 1993; R1) that the $V / R$ variations are stronger right before and during an outburst and best seen in the strong red He I lines. Therefore it is expected that a cloud of material has been ejected shortly before or at the beginning of the MuSiCoS 98 campaign and we see it orbiting around the star (see $V / R$ in Fig. 13) and going towards the already existing envelope (see peak separation in Fig. 12). After a few revolutions around the star, the cloud is not detected anymore.

Two schematic scenarios are proposed here to explain the $V / R$ variations at the beginning of the observing campaign and the large fluctuation observed between HJD 2451144.5 and 2451148. These scenarios need to be investigated further in the context of a global theoretical model.

Scenario A: a first compact cloud of material is ejected just prior to or at the beginning of the MuSiCoS campaign and is a precursor of a subsequent bigger ejection. After a few stellar rotational cycles, at HJD 2451147, this new material is ejected in an axisymmetrical way making the total emission stronger (Fig. 11). It dilutes the cloud, therefore the amplitude of the $V / R$ variations decreases (Fig. 13). The slow decrease of total emission observed after the outburst suggests that part of the ejected material falls back onto the star.

Scenario B: a compact cloud of material is ejected right before or at the beginning of the MuSiCoS campaign, and this is the only ejection. After a few rotational cycles, at HJD 2451147, the cloud reaches the already existing envelope and is circularized and diluted in the envelope, causing a decrease of the $V / R$ variations (see Fig. 13).
The emissivity of recombination lines is proportional to the density squared. Therefore the total emission of the cloud merged with the disk is higher than the summed emission of the cloud alone plus the disk alone (Fig. 11). This last statement is true only if the mass of the cloud is not negligible compared to the emitting mass of the disk. For $\omega$ Ori the mass of the cloud is not negligible, as we detect the $V / R$ variations it produces during the first part of the campaign. The slow decrease of total emission observed after the outburst suggests that when the dense cloud has been completely diluted in the disk, its contribution is not significant anymore, and/or the volume of the disk expanded because of the merging.

If the cloud of material seen before HJD 2451148 corotates with the star, $f_{2}=0.46 \mathrm{~cd}^{-1}$ would be the rotational frequency of $\omega$ Ori. However, we showed that $f_{\text {rot }}$ is around $0.73 \mathrm{c} \mathrm{d}^{-1}$ which implies that the cloud rotates slower than the star itself. If the cloud is in Keplerian orbit, using $R=6.8 R_{\odot}$ and $M=8.0 M_{\odot}$ determined in Sect. 2 , it is situated at a radius of $2.07 R_{*}$. The variation in the peak separation of $\mathrm{H} \alpha$ between HJD 2451147 and 48 indicates (Hanuschik et al. 1993) that, during the outburst, material was pushed out from a Keplerian orbit with radius $\sim 2.05 R_{*}$ up to $\sim 2.30 R_{*}$. This gives good confidence that the cloud was indeed pushed further away from the star at this period.

Taking extremes values for $R$ and $M$ (see Table 1), the lowest possible inner radius of the disk is not larger than the equatorial radius of the star. Therefore we cannot exclude that the disk is attached to the star. However, the extremes values of $R$ and $M$ are unlikely for such a star; the mean values of $R$ and $M$ are much more realistic and lead to a detached disk.

We stress the difficulty of finding a clear common definition of an outburst and its beginning. The word "outburst" has been used in the literature to describe a sudden enhancement of light or emission in lines. But it is also usually linked to an ejection of material, considered as the cause of the sudden light or emission increase.

In the case studied in this paper, an increase of emission occurred between HJD 2451147 and 2451148 and this is what we call here an outburst. It can be due to an ejection occuring at HJD 2451147 (scenario B) or to the merging of a cloud from a previous ejection with the disk (scenario A).

The time of beginning of the outburst depends on the line considered. For $\mu$ Cen (R1) observed that $\mathrm{H} \delta$ is the best line for determining the beginning of an outburst. This line was not observed as a good indicator for $\omega$ Ori, but following their paper, the beginning of the outburst in $\mathrm{H} \delta$ would be at HJD 2451148, as weak emission seems to affect this line between HJD 2451148 and 52 .

\section{Conclusions}

We have shown that the Be star $\omega$ Ori undergoes several variations. The star pulsates non-radially with a frequency of $1.03 \mathrm{~cd}^{-1}$ associated with a NRP mode with $l=2$ or 
3 and $|m|=2$. Some of the ejected material may still be linked to the star and pulsate as well. A second frequency is detected at $0.46 \mathrm{c} \mathrm{d}^{-1}$ and is attributed to material temporarily orbiting around the star ejected prior to or at the beginning of the campaign. Rotational modulation alone may be ruled out to explain all the observed variations. Both NRPs and rotational modulation of orbiting clouds are needed to explain short-term and mid-term variations in $\omega$ Ori.

We also showed that the cloud was diluted in the middle of the campaign when an outburst occurred, possibly enhanced by another ejection of material. A longer timebase is necessary to investigate whether these kinds of events could be periodic and attributed to beating effects of NRPs.

Acknowledgements. We wish to thank the MuSiCoS 98 team, including the technical staffs and time allocation committees for the telescopes. We are grateful to the French association of amateurs AUDE for providing $\mathrm{H} \alpha$ spectra, and to S. Brillant and N. V. Leister for the 2000/2001 NTT and FEROS data. We also thank the referee, D. Baade, for his constructive comments. This research has made use of the Simbad database maintained at CDS, Strasbourg, France.

\section{References}

Andrillat, Y., \& Fehrenbach, C. 1982, A\&AS, 48, 93

Baade, D. 1982, A\&A, 105, 65

Ballereau, D., Chauville, J., \& Zorec, J. 1995, A\&AS, 111, 423

Balona, L. A. 1986, MNRAS, 219, 111

Balona, L. A., Aerts, C., Božić, H., et al. 2001, MNRAS, 327, 1288 (B01)

Balona, L. A., Cuypers, J., \& Marang, F. 1992, A\&AS, 92, 533

Balona, L. A., \& Dziembowski, W. A. 1999, MNRAS, 309, 221 (B\&D)

Banerjee, D. P. K., Rawat, S. D., \& Janardhan, P. 2000, A\&AS, 147, 229

Bergin, E. A., Burns, J. F., Guinan, E. F., \& McCook, G. P. 1989, Informational Bulletin on Variable Stars, 3358, 1

Bopp, B. W., \& Dempsey, R. C. 1989, Informational Bulletin on Variable Stars, 3387, 1

Brown, A. G. A., de Geus, E. J., \& de Zeeuw, P. T. 1994, A\&A, 289, 101

Buil, C. 2001, Atlas of Be stars on the web http://www. astrosurf.com/buil/us/bestar.htm

Collins, G. W. 1987, in Physics of Be Stars, IAU Coll. 92, 3

Dachs, J., Eichendorf, W., Schleicher, H., et al. 1981, A\&AS, 43,427

Doazan, V., Sedmak, G., Barylak, M., \& Rusconi, L. 1991, ESA-SP 1147, 1147

Domiciano de Souza, A., Janot-Pacheco, E., Leister, N. V., et al. 2000, in The Be Phenomenon in Early-Type Stars, IAU Coll. 175, ed. M. A. Smith, H. F. Henrichs, \& J. Fabregat, ASP Conf. Ser., 214, 276

Domiciano de Souza Jr., A. 1999, Master's thesis, IAG-USP, Brazil

Donati, J.-F., Wade, G. A., Babel, J., et al. 2001, MNRAS, 326,1265

Duflot, M., Figon, P., \& Meyssonnier, N. 1995, A\&AS, 114, 269
Emilio, M. 1997, Master's Thesis, IAG-USP, Brazil

Floquet, M., Hubert, A. M., Hirata, R., et al. 2000a, A\&A, 362,1020

Floquet, M., Neiner, C., \& Hubert, A. M. 2000b, in The Be Phenomenon in Early-Type Stars, IAU Coll. 175, ed. M. A. Smith, H. F. Henrichs, \& J. Fabregat, ASP Conf. Ser., 214,260

Foster, G. 1995, AJ, 109, 1889

Foster, G. 1996, AJ, 111, 541

Gies, D. R. 1994, in Pulsation, Rotation and Mass Loss in Early-Type Stars, IAU Symp., 162, 89

Grady, C. A., Perez, M. R., Talavera, A., et al. 1996, A\&AS, 120,157

Gray, D. F. 1976, The observation and analysis of stellar photospheres (Wiley-Interscience)

Hanuschik, R. W., Dachs, J., Baudzus, M., \& Thimm, G. 1993, A\&A, 274, 356

Hanuschik, R. W., Hummel, W., Sutorius, E., Dietle, O., \& Thimm, G. 1996, A\&AS, 116, 309

Harmanec, P. 1982, in Be Stars, IAU Symp., 98, 279

Hayes, D. P., \& Guinan, E. F. 1984, ApJ, 279, 721

Henrichs, H. F., de Jong, J. A., Donati, J.-F., et al. 2000a, in The Be Phenomenon in Early-Type Stars, IAU Coll. 175, ed. M. A. Smith, H. F. Henrichs, \& J. Fabregat, ASP Conf. Ser., 214, 324

Henrichs, H. F., Neiner, C., Hubert, A. M., et al. 2000b, in The Be Phenomenon in Early-Type Stars, IAU Coll. 175, ed. M. A. Smith, H. F. Henrichs, \& J. Fabregat, ASP Conf. Ser., 214, 372

Hillenbrand, L. A., Strom, S. E., Vrba, F. J., \& Keene, J. 1992, ApJ, 397, 613

Hubert, A. M., \& Floquet, M. 1998, A\&A, 335, 565

Hubert, A. M., Floquet, M., Hao, J. X., et al. 1997, A\&A, 324, 929

Hubert-Delplace, A. M., \& Hubert, H. 1979, An atlas of Be stars (Paris-Meudon Observatory)

Jankov, S., Janot-Pacheco, E., \& Leister, N. V. 2000, ApJ, 540, 535

Kambe, E., Ando, H., \& Hirata, R. 1993a, A\&A, 273, 435

Kambe, E., Ando, H., Hirata, R., et al. 1993b, PASP, 105, 1222

Kennelly, E. J., Walker, G. A. H., Catala, C., et al. 1996, A\&A, 313,571

Kennelly, E. J., Walker, G. A. H., \& Merryfield, W. J. 1992, ApJ, 400, L71

Ledoux, P. 1951, ApJ, 114, 373

Marlborough, J. 1986, in Physics of Be Stars, IAU Coll., 92, 316

McDavid, D., Hirata, R., Guinan, E. F., Bjorkman, K. S., \& Babler, B. L. 1996, Amer. Astron. Soc. Meet., 28, 913

Mennickent, R. E., Vogt, N., Barrera, L. H., Covarrubias, R., \& Ramirez, A. 1994, A\&AS, 106, 427

Neiner, C., Hubert, A. M., Floquet, M., et al. 2002, in Radial and nonradial pulsations as probes of stellar physics, IAU Coll. 185, ed. C. Aerts, T. R. Bedding, \& J. ChristensenDalsgaard, ASP Conf. Ser., 259, 246

Osaki, Y. 1986, PASP, 98, 30

Oudmaijer, R. D., \& Drew, J. E. 1999, MNRAS, 305, 166

Perryman, M. A. C., Lindegren, L., Kovalevsky, J., et al. 1997, A\&A, 323, L49

Peters, G. J., \& Gies, D. R. 2000, in The Be Phenomenon in Early-Type Stars, IAU Coll. 175, ed. M. A. Smith, H. F. Henrichs, \& J. Fabregat, ASP Conf. Ser., 214, 375

Poeckert, R., \& Marlborough, J. M. 1976, ApJ, 206, 182 
Quirrenbach, A., Bjorkman, K. S., Bjorkman, J. E., et al. 1997, Thé, P. S., de Winter, D., \& Perez, M. R. 1994, A\&AS, 104, ApJ, 479, 477 315

Rivinius, T., Baade, D., Stefl, S., et al. 1998a, A\&A, 333, $125(\mathrm{R} 1)$

Rivinius, T., Baade, D., Stefl, S., et al. 1998b, A\&A, 336, 177

Townsend, R. 2000, in The Be Phenomenon in Early-Type Stars, IAU Coll. 175, ed. M. A. Smith, H. F. Henrichs, \& J. Fabregat, ASP Conf. Ser., 214, 288

Rivinius, T., Baade, D., Stefl, S., et al. 1998c, in Cyclical Variability in Stellar Winds, 207

Rivinius, T., Baade, D., Štefl, S., et al. 2001, A\&A, 369, 1058

Schaller, G., Schaerer, D., Meynet, G., \& Maeder, A. 1992, A\&AS, 96, 269

Smith, M. A. 1989, ApJS, 71, 357

Sonneborn, G., Grady, C. A., Wu, C., et al. 1988, ApJ, 325, 784

Srinivasan, K. 1996, Ph.D. Thesis, Bharathidasan University

Stee, P., \& Bittar, J. 2001, A\&A, 367, 532

Telting, J. H., \& Schrijvers, C. 1997, A\&A, 317, 723 (T\&S)

Underhill, A. B. 1987, in Physics of Be Stars, IAU Coll., 92, 411

Štefl, S., Budovičová, A., Baade, D., et al. 2000, in The Be Phenomenon in Early-Type Stars, ASP Conf. Ser., 214, 240

Zinnecker, H., \& Preibisch, T. 1994, A\&A, 292, 152

Zorec, J., \& Briot, D. 1991, A\&A, 245, 150

Zorec, J., Frémat, Y., Hubert, A. M., \& Floquet, M. 2002, in Radial a nd nonradial pulsations as probes of stellar physics, IAU Coll. 185, ed. C. Aerts, T. R. Bedding, \& J. Christensen-Dalsgaard, ASP Conf. Ser., 259, 244 